

ANL/NE-11/25

\title{
CFD Validation in OECD/NEA T-Junction Benchmark
}

Mathematics and Computer Science Division 


\begin{abstract}
About Argonne National Laboratory
Argonne is a U.S. Department of Energy laboratory managed by UChicago Argonne, LLC under contract DE-AC02-06CH11357. The Laboratory's main facility is outside Chicago, at 9700 South Cass Avenue, Argonne, Illinois 60439. For information about Argonne and its pioneering science and technology programs, see www.anl.gov.
\end{abstract}

\title{
Availability of This Report
}

This report is available, at no cost, at http://www.osti.gov/bridge. It is also available on paper to the U.S. Department of Energy and its contractors, for a processing fee, from:

U.S. Department of Energy

Office of Scientific and Technical Information

P.O. Box 62

Oak Ridge, TN 37831-0062

phone (865) 576-8401

fax (865) 576-5728

reports@adonis.osti.gov

\begin{abstract}
Disclaimer
This report was prepared as an account of work sponsored by an agency of the United States Government. Neither the United States Government nor any agency thereof, nor UChicago Argonne, LLC, nor any of their employees or officers, makes any warranty, express or implied, or assumes any legal liability or responsibility for the accuracy, completeness, or usefulness of any information, apparatus, product, or process disclosed, or represents that its use would not infringe privately owned rights. Reference herein to any specific commercial product, process, or service by trade name, trademark, manufacturer, or otherwise, does not necessarily constitute or imply its endorsement, recommendation, or favoring by the United States Government or any agency thereof. The views and opinions of document authors expressed herein do not necessarily state or reflect those of the United States Government or any agency thereof, Argonne National Laboratory, or UChicago Argonne, LLC.
\end{abstract}




\section{CFD Validation in OECD/NEA T-Junction Benchmark}

prepared by

A.V. Obabko, ${ }^{1}$ P.F. Fischer, and T.J. Tautges ${ }^{2}$

Mathematics and Computer Science Division

Argonne National Laboratory, Argonne, IL 60439, USA

\section{S. Karabasov}

Cambridge University, Cambridge, UK

V.M. Goloviznin and M.A. Zaytsev, ${ }^{3}$

V.V. Chudanov, V.A. Pervichko, and A.E. Aksenova

Moscow Institute of Nuclear Energy Safety (IBRAE),

Moscow, Russia

1'obabko@mcs.anl.gov

${ }^{2}$ also from Univ. of Wisconsin, Madison, WI, USA

${ }^{3}$ also from Russian Academy of Science (RAS), Moscow, Russia

June 2011 


\section{Contents}

$\begin{array}{ll}\text { Abstract } & 1\end{array}$

1 Introduction 1

2 Experimental Configuration 2

3 Nek5000 Simulations $\quad 3$

4 Cabaret Simulations 5

5 Conv3D Simulations $\quad 8$

6 Results $\quad 10$

6.1 Comparison with Experiment . . . . . . . . . . . . . . . . 10

6.1 .1 Cold and Hot Inlets . . . . . . . . . . . . . . . . . . . . . . . . 10

6.1.2 Downstream of the T-Junction . . . . . . . . . . . . . . . 13

6.2 Sensitivity Study . . . . . . . . . . . . . . . . . . . . 15

6.3 Study of Reversed Flow Region . . . . . . . . . . . . . . . . . 17

7 Conclusions and Future Work $\quad 18$

$\begin{array}{ll}\text { Acknowledgments } & 19\end{array}$

$\begin{array}{ll}\text { References } & 19\end{array}$ 


\title{
CFD Validation in OECD/NEA T-Junction Benchmark
}

\author{
Aleksandr V. Obabko, ${ }^{\mathrm{a}}$ Paul F. Fischer, and Timothy J. Tautges ${ }^{\mathrm{b}}$ \\ Mathematics and Computer Science Division \\ Argonne National Laboratory, Argonne, IL 60439, U.S.A. \\ Sergey Karabasov \\ Cambridge University \\ Cambridge, U.K. \\ Vasily M. Goloviznin and Mikhail A. Zaytsev \\ Vladimir V. Chudanov, Valeriy A. Pervichko, and Anna E. Aksenova \\ Moscow Institute of Nuclear Energy Safety (IBRAE) \\ Moscow, Russia \\ a obabko@mcs.anl.gov \\ ${ }^{\mathrm{b}}$ Engineering Physics Department \\ University of Wisconsin, Madison, WI 53706, U.S.A. \\ ${ }^{\mathrm{c}}$ Russian Academy of Science (RAS) \\ Moscow, Russia
}

\begin{abstract}
We compare and contrast simulation results for three computational fluid dynamics codes CABARET, Conv3D, and Nek5000 for the T-junction thermal striping problem that was the focus of a recent OECD/NEA blind benchmark. The corresponding codes utilize finite-difference implicit large eddy simulation (ILES), finite-volume LES on fully staggered grids, and an LES spectral element method (SEM), respectively. The simulations results are in a good agreement with experimenatl data. We present results from a study of sensitivity to computational mesh and time integration interval, and discuss the next steps in the simulation of this problem.
\end{abstract}

\section{Introduction}

When streams of rapidly moving flow merge in a T-junction, the potential arises for large oscillations at the scale of the diameter, $D$, with a period scaling as $O(D / U)$, where $U$ is the characteristic flow velocity (see Figure 1). If the streams are of different temperatures, the oscillations result in experimental fluctuations (thermal striping) at the pipe wall in the outlet branch that can accelerate thermal-mechanical fatigue and ultimately cause pipe failure. The importance of this phenomenon has prompted the nuclear energy 


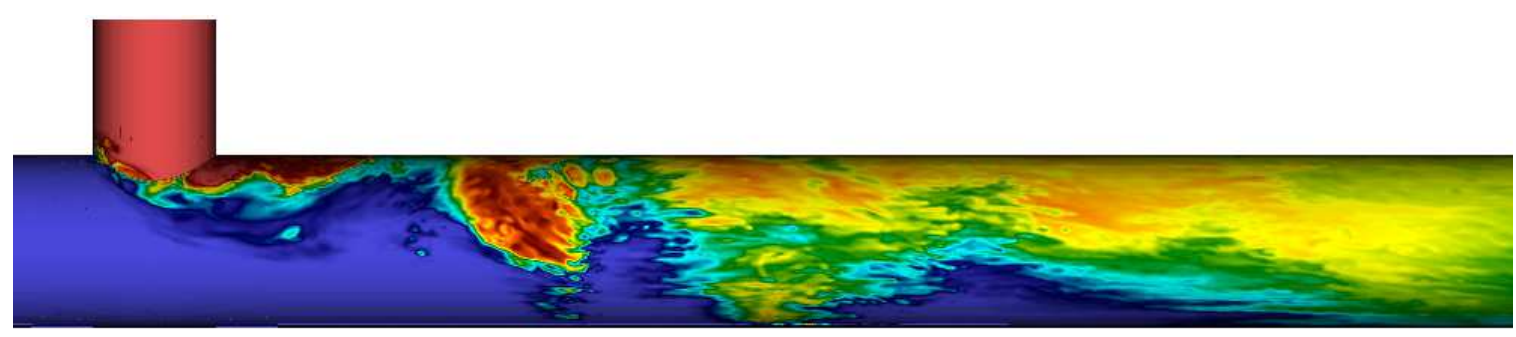

Figure 1. Instantaneous wall temperature distribution from Nek5000 T-junction simulation.

modeling and simulation community to establish a benchmark to test the ability of computational fluid dynamics (CFD) codes to predict thermal striping. The benchmark is based on thermal and velocity data measured in an experiment designed specifically for this purpose $[19,22]$. Thermal striping is intrinsically unsteady and hence not accessible to steady state simulation approaches such as steady state Reynolds-averaged Navier-Stokes (RANS) models. ${ }^{1}$ Consequently, one must consider either unsteady RANS or large eddy simulation (LES). This report compares the results for three LES codes: Nek5000, developed at Argonne National Laboratory (USA), and CABARET and Conv3D, developed at the Moscow Institute of Nuclear Energy Safety at (IBRAE) in Russia. Nek5000 is based on the spectral element method (SEM), which is a high-order weighted residual technique that combines the geometric flexibility of the finite element method (FEM) with the tensor-product efficiencies of spectral methods [20,6]. CABARET is a "compact accurately boundary-adjusting high-resolution technique" for fluid dynamics simulation [15]. The method is second-order accurate on nonuniform grids in space and time, and has a small dispersion error and computational stencil defined within one space-time cell. The scheme is equipped with a conservative nonlinear correction procedure based on the maximum principle. CONV3D is based on the immersed boundary method and is validated on a wide set of the experimental and benchmark data. The numerical scheme has a very small scheme diffusion and is the second and the first order accurate in space and time, correspondingly.

This report is organized as follows. In Section 2 we describe the experimental setup and data collection procedure. In Section 3-5 we outline the computational models. The results are discussed in Section 6, including a comparison of data from the simulations and experiment (Section 6.1). Concluding remarks are provided in Section 7.

\section{Experimental Configuration}

The Vatenfall experiment [26] is based on water flow in a main pipe of diameter $D=140$ $\mathrm{mm}$ with a side branch of diameter $D_{H}=100 \mathrm{~mm}$ adjoining the main at a 90 degree angle. The pipes and the T-junction, which is made from a plexiglass block, are transparent so that the velocity can be measured with laser Doppler anemometry (LDA). Velocity data

\footnotetext{
${ }^{1}$ The performance of steady state RANS coupled with stability computations e.g., with Parabolized Stability Equations has to be further investigated.
} 
Table 1

Dimensional Parameters for Vatenfall T-junction Experiment

\begin{tabular}{|l|c|c|}
\hline Parameter & Main Branch & Hot Branch \\
\hline Diameter $D^{*}, D_{H}^{*}(\mathrm{~m})$ & 0.1400 & 0.1000 \\
Flow rate $q^{*}, q_{H}^{*}(\mathrm{l} / \mathrm{s})$ & 9.00 & 6.00 \\
Average velocity $U^{*}, U^{*}{ }_{H}(\mathrm{~m} / \mathrm{s})$ & 0.585 & 0.764 \\
Inlet temperature $\left({ }^{\circ} \mathrm{C}\right)$ & 19.0 & 36.0 \\
Density $\rho\left(\mathrm{kg} / \mathrm{m}^{3}\right)$ & 998.5 & 993.7 \\
Dynamic viscosity $\left(N s / \mathrm{m}^{3}\right)$ & $1.029 \mathrm{e}-3$ & $7.063 \mathrm{e}-4$ \\
Kinematic viscosity $\nu\left(\mathrm{m}^{2} / \mathrm{s}\right)$ & $1.031 \mathrm{e}-6$ & $7.108 \mathrm{e}-7$ \\
\hline
\end{tabular}

Table 2

Nondimensional Parameters for Vatenfall T-junction Experiment

\begin{tabular}{|l|c|c|}
\hline Parameter & Main Branch & Hot Branch \\
\hline Diameter $D, D_{H}$ & 1.000 & 0.714 \\
Average velocity $U, U_{H}$ & 1.000 & 1.307 \\
Inlet temperature & 0.000 & 1.000 \\
Density & 1.000 & 0.9952 \\
Reynolds number $R e, R e_{H}$ & 79400 & 107000 \\
\hline
\end{tabular}

was taken under isothermal conditions with both flows entering at $19{ }^{\circ} \mathrm{C}$. In order to measure thermal striping, time dependent temperature data was collected from thermocouples downstream of the T-junction with flow at $19{ }^{\circ} \mathrm{C}$ entering the main branch and flow at $36^{\circ} \mathrm{C}$ entering the side branch.

The flow enters the cold (main) branch from a stagnation chamber located $80 \mathrm{D}$ upstream of the T-junction and is assumed to be fully developed turbulent flow by the time it reaches the T-junction. The hot branch flow enters at $20 D_{H}$ upstream and is not quite fully developed as it enters the T-junction. The inlet flow rates are 9 and $6 \mathrm{l} / \mathrm{s}$ (liters per second), respectively in the cold and hot branches, which corresponds to a Reynolds number of $R e \approx 80,000$ and 100,000 , respectively. The key dimensional parameters are summarized in Table 1 and their nondimensional counterparts in Table 2.

\section{Nek5000 Simulations}

The Nek5000 simulations are based on the spectral element method developed by Patera [20]. Nek5000 supports two formulations for spatial and temporal discretization of the Navier-Stokes equations. The first is the $\mathbb{P}_{N}-\mathbb{P}_{N-2}$ method $[18,10,9]$, in which the velocity/pressure spaces are based on tensor-product polynomials of degree $N$ and $N-2$, respectively, in the reference element $\hat{\Omega}:=[-1,1]^{d}$, for $d=2$ or 3 . The computational domain consists of the union of $E$ elements $\Omega^{e}$, each of which is parametrically mapped from $\hat{\Omega}$ to yield a body-fitted mesh. The second is the low-Mach number formulation due to Tomboulides and Orszag [24,25], which uses consistent order- $N$ approximation spaces for both the velocity and pressure. The low-Mach number formulation is also valid in the 
(a)

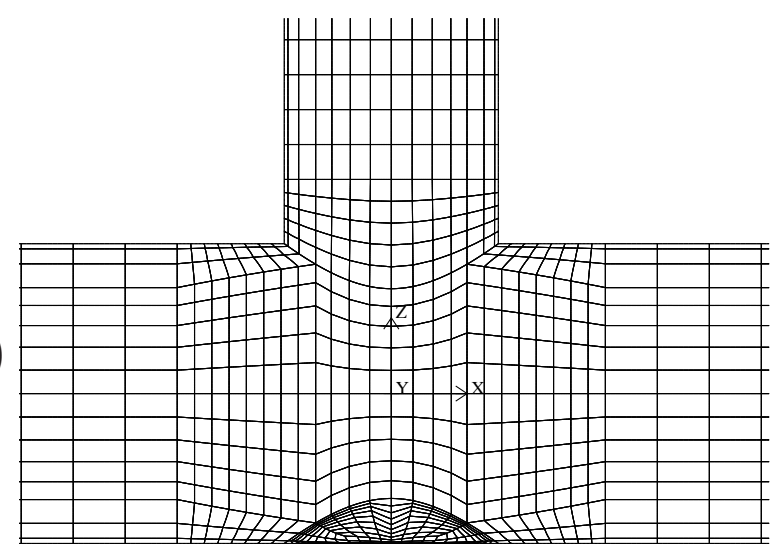

(b)

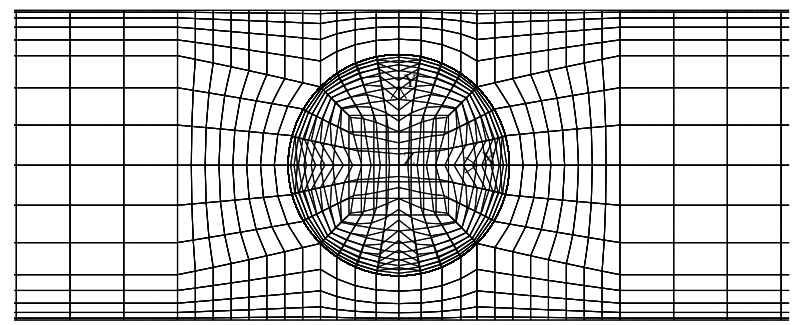

(c)

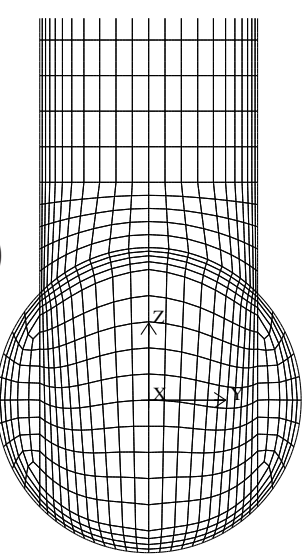

(d)

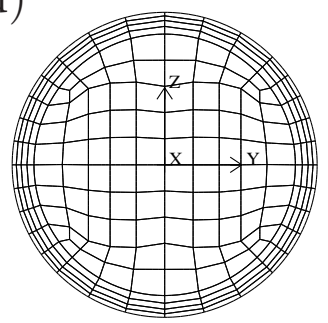

Figure 2. Computational mesh for Nek5000 T-junction simulation comprising $E=62176$ elements: (a) side view of surface mesh, (b) top view, (c) slice at $x=0$, and (d) slice at $x=4$.

zero-Mach (incompressible) limit [23]. Both formulations yield a decoupled set of elliptic problems to be solved at each timestep. In $d=3$ space dimensions, one has three Helmholtz solves of the form $(\beta I+\nu \Delta t A) \underline{u}_{i}^{n}=\underline{f}_{i}^{n}, i=1, \ldots, d$, and a pressure Poisson solve of the form $A \underline{p}^{n}=g^{n}$ at each timestep, $t^{n}, \underline{n}=1, \ldots$ Here, $A$ is the symmetric positive definite Laplace operator, and $\beta$ is an order-unity coefficient coming from a 3rd-order backwarddifference approximation to the temporal derivative. (For the $\mathbb{P}_{N}-\mathbb{P}_{N-2}$ method, the Laplace operator $A$ is replaced by a spectrally equivalent matrix arising from the unsteady Stokes equations $[9,6]$.) For marginally-resolved LES cases, we find that the higher-order pressure approximation of the $\mathbb{P}_{N}-\mathbb{P}_{N}$ formulation tends to yield improved skin-friction estimates, and this is consequently the formulation considered here.

The computational mesh for the Nek5000 simulations was generated by using CUBIT and consists of $E=62,176$ elements. Within each element, velocity and pressure are represented as Lagrange interpolating polynomials on tensor products of $N$ th-order GaussLobatto-Legendre (GLL) points. Unless otherwise noted, all the simulations were run with polynomial order $N=7$, corresponding to a total number of mesh points $n \approx E N^{3} \approx 21$ million. Figure 2 shows a closeup of the mesh in the vicinity of the origin, which is located at the intersection of the branch centerlines. The inlet for the main branch is at 
$x=-9.2143$ and for the hot branch at $z=6.4286$. These lengths permitted generation of fully-developed turbulence upstream of the T-junction, as described below. The outlet at $x=22$ was chosen to allow downstream tracking of temperature data at locations provided in the experiment. Away from the origin, the axial extent of the spectral elements in the main branch is $0.18 D$, corresponding to a maximum axial mesh spacing of $\delta x_{\max }=0.0377$. At the wall, the wall-normal element size is 0.01222 , corresponding to a minimum spacing of $\delta y_{n} \approx 0.0008$. The submitted simulations were run with $R e=40,000$ in the inlet branches, yielding $R e=60,000$ in the outlet. Downstream of the T-junction, the first grid point away from the wall is thus at $y^{+} \approx 2.5$ in wall units.

Inlet flow conditions in the main branch are based on a recycling technique in which the inlet velocity at time $t^{n}$ is given by $\alpha \mathbf{u}\left(\tilde{x}, y, z, t^{n-1}\right)$, where $\tilde{x}=-3$ and $\alpha$ is chosen to ensure that the mass flow rate at inflow is constant $\left(\int u^{n}(-9, y, z) d y d z \equiv \pi\right)$. Recycling is also used for the hot branch, save that the inflow condition is $0.8 \beta \mathbf{u}\left(x, y, \tilde{z}, t^{n-1}\right)-0.2 U_{H}$, with $\beta$ chosen so that the average inflow velocity is $-U_{H}$ and $\tilde{z}=2.1$. The 0.8 multiplier was added in order to give a flatter profile characteristic of the non-fully-developed flow realized in the experiment, but a systematic study of this parameter choice was not performed.

Initial conditions for all branches were taken from fully-developed turbulent pipe flow simulations at $R e=40,000$. The timestep size was $\Delta t=2.5 \times 10^{-4}$ in convective time units, or about $6 \times 10^{-5}$ seconds in physical units corresponding to the experiment. The simulation was run to a time of $t=28$ convective time units prior to acquiring data. Data was then collected over the interval $t \in[28,58]$ (in convective time units) for the benchmark submission and over $t \in[28,104]$ subsequently (longer average) both with $N=7$. In addition to $N=7$ results (i.e., with $n \approx E N^{3} \approx 2.1 \times 10^{7}$ points), Nek5000 runs were conducted with $N=5\left(n \approx 7.7 \times 10^{6}\right.$ points $)$. These runs started with the $N=7$ results and were run and averaged over about 110 convective time units (i.e., about 26 seconds). The timestep size for $N=5$ runs was twice as big (i.e, $\Delta t=5 \times 10^{-4}$ ). Note that all Nek5000 results reported here were obtained with constant density equal to the nondimensional value of 1.000 (see Table 2).

\section{Cabaret Simulations}

The system of Navier-Stokes equations in a slightly compressible form (i.e., for a constant sound speed) is solved with a new code based on the Compact Accurately Adjusting high-REsolution Technique (CABARET). In order to lessen the computational grid requirements, the code uses hybrid unstructured hexahedral/tetrahedral grids. CABARET is extension of the original second-order upwind leapfrog scheme [14] to nonlinear conservation laws $[21,11]$ and to multiple dimensions $[12,17]$. In summary, CABARET is an explicit nondissipative conservative finite-difference scheme of second-order approximation in space and time. In addition to having low numerical dispersion, CABARET has a very compact computational stencil due to the use of separate variables for fluxes and conservation. The stencil is staggered in space and time and for advection includes only one computational cell. For nonlinear flows, CABARET uses a low-dissipative conservative flux correction method directly based on the maximum principle [15]. In the LES framework, the non-linear flux correction plays the role of implicit turbulence closure 
following the MILES approach of Grinstein and Fureby [13, ch.5] which was discussed in the ocean modeling context in [16].

A detailed description of the CABARET code on a mixed unstructured grid will be the subject of a future publication; an outline of the method on a structured Cartesian grid is given below. Let us consider the governing equations written in the standard conservation form:

$$
\frac{\partial \mathbf{U}}{\partial t}+\frac{\partial \mathbf{F}}{\partial x}+\frac{\partial \mathbf{G}}{\partial y}+\frac{\partial \mathbf{W}}{\partial z}=\mathbf{Q}_{\nu}
$$

where the sources in the right-hand side include viscous terms. By mapping the physical domain to the grid space-time coordinates $(x, y, z, t) \rightarrow(i, j, k, n)$ and referring control volumes to the cell centers (fractional indices) and fluxes to the cell faces (integer indices), the algorithm proceeds from the known solution at time level (n) to the next timestep $(\mathrm{n}+1)$ as follows:

- Conservation predictor step:

$$
\frac{\mathbf{U}_{i+\frac{1}{2}, j+\frac{1}{2}, k+\frac{1}{2}}^{n+\frac{1}{2}}-\mathbf{U}_{i+\frac{1}{2}, j+\frac{1}{2}, k+\frac{1}{2}}^{n}}{0.5 t}+\frac{\mathbf{F}_{i+1}^{n}-\mathbf{F}_{i}^{n}}{\Delta x}+\frac{\mathbf{G}_{j+1}^{n}-\mathbf{G}_{j}^{n}}{\Delta y}+\frac{\mathbf{W}_{k+1}^{n}-\mathbf{W}_{k}^{n}}{\Delta z}=\mathbf{Q}_{\nu}^{n}
$$

- Upwind extrapolation based on the characteristic decomposition:

- For each cell face, decompose the conservation and flux variables into local Riemann fields, $\mathbf{U} \rightarrow R_{q}, q=1, \ldots, N$, that correspond to the local cell-facenormal coordinate basis, where $N$ is the dimension of the system.

- For each cell face at the new timestep, compute a dual set of preliminary local Riemann variables that correspond to the upwind and downwind extrapolation of the characteristic fields, e.g., $\tilde{R}_{q}^{n+1}=\left(2 R_{q}^{n+\frac{1}{2}}\right)_{\text {upwind/downwind cell }}-\left(R_{q}^{n}\right)_{\text {local face }}$ for the upwind/downwind conservation volumes.

- Correct the characteristic flux fields if they lie outside the monotonicity bounds $\left(R_{q}^{n+1}\right)=\max \left(R_{q}\right)^{n}, \tilde{R}_{q}^{n+1}>\max \left(R_{q}\right)^{n} ;\left(R_{q}^{n+1}\right)=\min \left(R_{q}\right)^{n}, \tilde{R}_{q}^{n+1}<\min \left(R_{q}\right)^{n} ;$ else $\left(R_{q}^{n+1}\right)=\tilde{R}_{q}^{n+1}$.

For reconstructing a single set of flux variables at the cell face, use an approximate Riemann solver.

- Conservation corrector step:

$$
\frac{\mathbf{U}_{i+\frac{1}{2}, j+\frac{1}{2}, k+\frac{1}{2}}^{n+1}-\mathbf{U}_{i+\frac{1}{2}, j+\frac{1}{2}, k+\frac{1}{2}}^{n+\frac{1}{2}}}{0.5 t}+\frac{\mathbf{F}_{i+1}^{n+1}-\mathbf{F}_{i}^{n+1}}{\Delta x}+\frac{\mathbf{G}_{j+1}^{n+1}-\mathbf{G}_{j}^{n+1}}{\Delta y}+\frac{\mathbf{W}_{k+1}^{n+1}-\mathbf{W}_{k}^{n+1}}{\Delta z}=2 \mathbf{Q}_{\nu}^{n+\frac{1}{2}}-\mathbf{Q}_{\nu}^{n}
$$

where a second-order central approximation is used for the viscous term. 

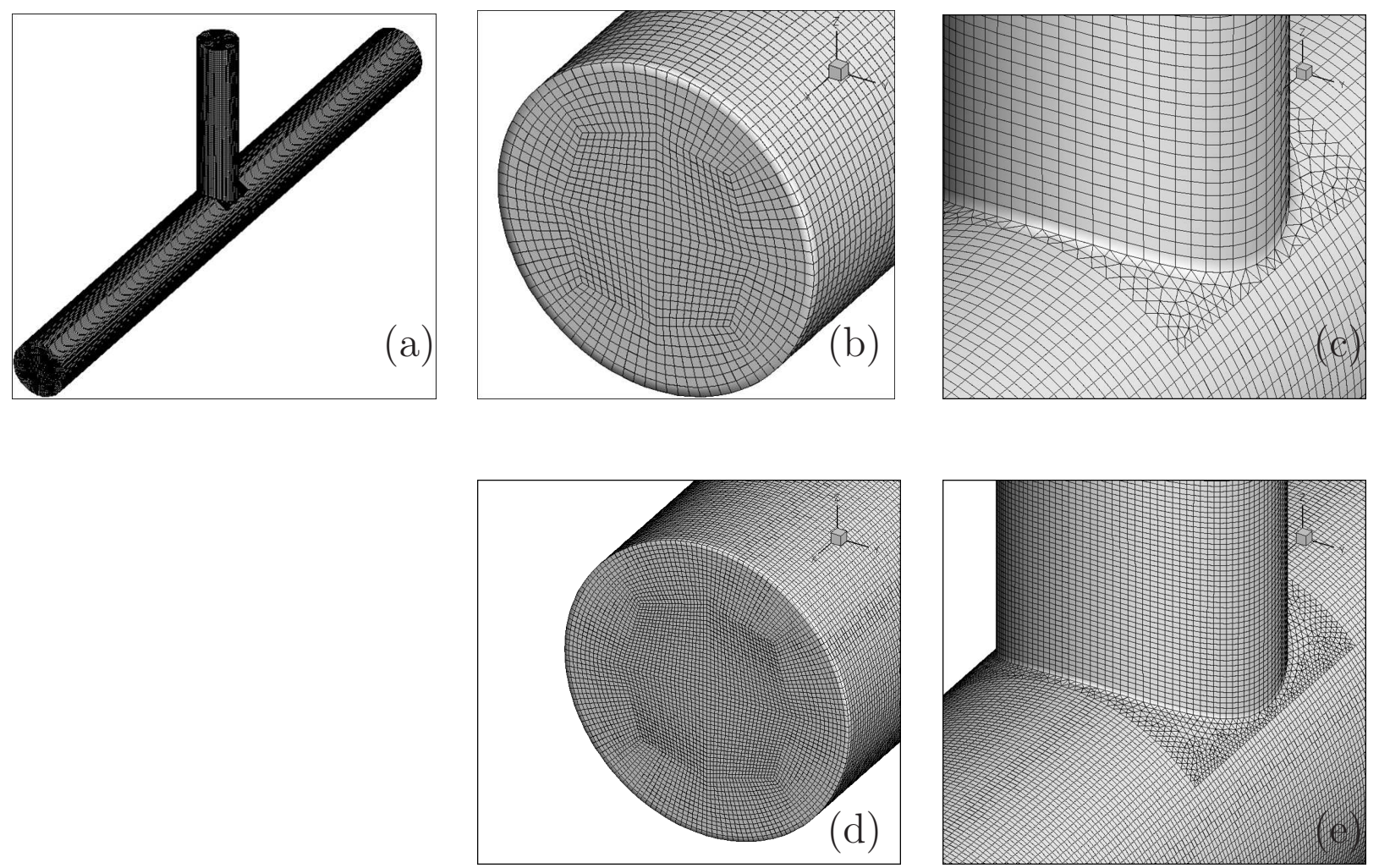

Figure 3. Computational grid used with the CABARET method: full domaim (a), pipe inlet $(b, d)$ and mixed grid elements in the vicinity of the junction $(\mathrm{c}, \mathrm{e})$ for the 0.5 and 4 million cell grid, respectively.

For the T-junction problem with the CABARET method, two hybrid computational grids are used with 0.53 and 4 million cells. Figure 3 shows the layout of (a) the computational domain, $(\mathrm{b}, \mathrm{d})$ the hexahedral grid with a uniform Cartesian block in the pipe centre, and (c,e) a small collar area of the pipe junction covered by the mixed hexahedral/tetrahedral elements for the smaller and larger grid, respectively.

For specifying inlet boundary conditions, a recycling technique is used similar to that for Nek5000. The outflow boundary is prescribed by using characteristic boundary conditions. For the inlet boundaries at the main and the hot branch, laminar inflow conditions are specified based on prescribing the mean flow velocity profiles. The length of the pipe upstream of the junction was sufficiently far from the junction to permit an adequate turbulent flow upstream of the junction. The outflow boundary is imposed at 20 jet diameters downstream of the junction, where characteristic boundary conditions are set.

In order to speed statistical convergence, the LES CABARET solution was started from a precursor RANS k-epsilon calculation. The CABARET simulation was then run for 10 seconds to allow the statistics to settle down. This was followed by the production run during which the required solution fields were stored for a duration of 5 and 10 seconds. 


\section{Conv3D Simulations}

Researches at IBRAE have been developing 3D unified numerical thermal hydraulic technique for safety analysis of the nuclear power plants, which includes (1) methods, algorithms, and software for automatically generating computing grids with local refinement near body borders, (2) methods and algorithms for solving of heat and mass transfer compressible/incompressible problems for research of 3D thermal hydraulic phenomena, and (3) approaches for modeling of turbulence.

For grid construction in IBRAE an automatic technology using CAD systems for designing of computational domain has been developed. A generation of structured orthogonal/Cartesian grids with a local refinement near boundaries is incorporated into a specially developed program that has a user-friendly interface and can be utilized on parallel computers [2]. The computational technique is based on the developed algorithms with small-scheme diffusion, for which discrete approximations are constructed with the use of finite-volume methods and fully staggered grids. For modeling 3D turbulent single-phase flows, the LES approach (commutative filters) and a quasi-direct numerical simulation (QDNS) approach are used. For simulation of 3D turbulent two-phase flows by means of DNS, detailed grids and effective numerical methods developed in IBRAE for solving CFD problems are applied. For observing the interface of two-phase flow, a modified level set (LS) method and multidimensional advection/convection schemas of total variation diminishing (TVD) type with small scheme diffusion involving sub-grid simulation (with local resolution) are used. The Conv3D code is fully parallelized and highly effective at the high performance computers. The developed modules were validated on a series of well-known tests with Rayleigh numbers ranging from $10^{6}$ to $10^{16}$ and Reynolds numbers ranging from $10^{3}$ to $10^{5}$.

In oder to simulate thermal hydraulic phenomena in incompressible media, the timedependent incompressible Navier-Stokes equations in the primitive variables [1] coupled with the energy equation are used:

$$
\begin{aligned}
& \frac{d \vec{v}}{d t}=-\operatorname{grad} p+\operatorname{div} \frac{\mu}{\rho} \operatorname{grad} \vec{v}+g, \\
& \operatorname{div} \vec{v}=0 \\
& \frac{\partial h}{\partial t}+\operatorname{div}(\vec{v} h)=\operatorname{div}\left(\frac{k}{\rho} \operatorname{grad} T\right), \\
& h=\int_{0}^{T} c(\xi) d \xi,
\end{aligned}
$$

where $p$ is pressure, normalized by the density. The basic features of the numerical algorithm [3,4] are the following. An operator-splitting scheme for the Navier-Stokes equations is used as the predictor-corrector procedure with correction for the pressure $\delta p$ :

$$
\begin{aligned}
& \frac{v^{n+1 / 2}-v^{n}}{\tau}+\left(C(v)-\operatorname{div} \frac{\mu}{\rho} \operatorname{grad}\right) v^{n+1 / 2}+\operatorname{grad} p^{n}-g, \\
& \operatorname{div}_{h}\left(\frac{1}{\rho} \operatorname{grad}_{h} \delta p^{n+1}\right)=\frac{1}{\tau} \operatorname{div}_{h} v^{n+1 / 2},
\end{aligned}
$$




$$
v^{n+1}=v^{n+1 / 2}-\frac{\tau}{\rho} \operatorname{grad}_{h} \delta p^{n+1} .
$$

In order to construct the time-integration scheme for the energy equation, its operators are decomposed into two parts associated with the enthalpy and temperature, respectively, that results in the following two-step procedure:

$$
\begin{aligned}
& \frac{h^{n+1 / 2}-h^{n}}{\tau}+\tilde{C}\left(u^{n}\right) h^{n+1 / 2}=0, \\
& \frac{h^{n+1}-h^{n+1 / 2}}{\tau}-\tilde{N} T^{n+1}=0 .
\end{aligned}
$$

In the momentum equation, the operators are also split into two parts. The first part is associated with the velocity transport by convection/diffusion written in linearized form as $A_{1}=C\left(u^{n}\right)+N$, where $N=\operatorname{div}\left(\frac{\mu}{\rho} \operatorname{grad} v\right)$. The second part deals with the pressure gradient $A_{2}=$ grad. We note that the gradient and divergence operators are adjoints of each other (i.e., $A_{2}^{*}=-$ div). The additive scheme of splitting looks like the following:

$$
\begin{aligned}
& \frac{v^{n+1 / 2}-v^{n}}{\tau}+A_{1} v^{n+1 / 2}+A_{2} p^{n}=f^{n}, \\
& \frac{v^{n+1}-v^{n}}{\tau}+A_{1} v^{n+1 / 2}+A_{2} p^{n+1}=f^{n}, \\
& A_{2}^{*} v^{n+1}=0,
\end{aligned}
$$

where $f^{n}$ is the right-hand side. This numerical scheme is used as the predictor-corrector procedure. That is, introducing the pressure correction in Equations 14-15 leads to the well-known Poisson equation and the equation for velocity correction in the form of Equations 9-10.

In computational mathematics there are recognized two variants of fictitious domain methods: continuation of coefficients at lower-order derivatives and continuation of coefficients at the highest-order derivatives. Both approaches are commonly used in computational fluid dynamics involving phase change processes. Here the first variant is employed, which in a physical sense can be considered as inclusion into the momentum equations of the model of a porous medium:

$$
\begin{aligned}
& \frac{\partial v_{\epsilon}}{\partial \tau}+\tilde{N}\left(v_{\epsilon}\right) v_{\epsilon}-\operatorname{div}\left(\frac{\mu}{\rho} \operatorname{grad} v_{\epsilon}\right)+\operatorname{grad} p+c_{\epsilon} v_{\epsilon}=f_{\epsilon}, \\
& \operatorname{div} v_{\epsilon}=0 .
\end{aligned}
$$

Various formulae of $c_{\epsilon}$ can be employed for the flow resistance term in these equations. For Equations 16-17, the modified predictor-corrector procedure taking into account the fictitious domain method looks like the following:

$$
\begin{aligned}
& \frac{v_{\epsilon}^{n+1 / 2}-v_{\epsilon}^{n}}{\tau}+A_{1} v_{\epsilon}^{n+1 / 2}+A_{2} p_{\epsilon}^{n}+c_{\epsilon} v_{\epsilon}^{n+1 / 2}=f^{n}, \\
& \operatorname{div}_{h}\left(\frac{1}{\rho} \operatorname{grad}_{h} \delta p^{s+1}\right)=\operatorname{div}_{h}\left(\frac{1}{\rho} \frac{\tau c_{\epsilon}}{1+\tau c_{\epsilon}} \operatorname{grad}_{h} \delta p^{s}\right)+\frac{1}{\tau} \operatorname{div}_{h} v_{\epsilon}^{n+1 / 2},
\end{aligned}
$$




$$
v_{\epsilon}^{n+1}=v_{\epsilon}^{n+1 / 2}-\frac{1}{\rho} \frac{1}{1+\tau c_{\epsilon}} \operatorname{grad}_{h} \delta p_{\epsilon} .
$$

An iterative method with a Chebyshev set of parameters using an FFT solver for Laplace operator as a preconditioner can serve as an alternative to conjugate gradient method. The application of this approach for solving of the elliptical equations with variable coefficients allows one to reach 50 times the acceleration of the commonly used method of conjugate gradients. For solving the convection problem, a regularized nonlinear monotonic operator-splitting scheme was developed [8]. A special treatment of approximation of convection terms $C(v)$ results in the discrete convective operator, which is skew-symmetric and does not contribute to the kinetic energy (i.e., is energetically neutral [8]). The numerical scheme is the second and the first order accurate in space and time, correspondingly. The algorithm is stable at a large enough integration timestep. Details of the validation for the presented approach on a wide set of both 2D and 3D tests are reported in [5].

In this report, we present the numerical results computed with Conv3D on a uniform mesh with 40 million nodes. For sensitivity study (Section 6.2), we provide results from computation on a uniform mesh with 12 million nodes and on a nonuniform mesh with 3 and 40 million nodes with near-wall refinement.

\section{Results}

We focus here on a comparison of the experimental data with the numerical results from three simulation codes: Nek5000, CABARET, and Conv3D. In Section 6.2 we study the velocity field sensitivity to the computational mesh and integration time interval. A similar sensitivity study is undertaken in Section 6.3 where we investigate the effects of grid resolution and time integration interval on the reattachment region immediately downstream of T-junction. A comparison of velocity and temperature spectra for these codes will be presented separately in a supplementary report [7].

All results presented here are nondimensionalized with the cold inlet parameters according to Table 1. For reference, the mean and rms quantities for a set of temporal values $u_{n}=u\left(t=t_{n}\right), n=1, \ldots, M$, are defined as usual:

$$
u=\frac{1}{M} \sum_{n=1}^{M} u_{n}, \quad u^{\prime}=\sqrt{\frac{1}{M} \sum_{n=1}^{M}\left(u_{n}-u\right)^{2}} .
$$

\subsection{Comparison with Experiment}

In this section, we compare the results of three codes with experimental data. First we look at the inlet profiles in the cold and hot branches of the T-junction and then at the vertical and horizontal profiles of the mean and rms axial velocity downstream of the junction.

\subsubsection{Cold and Hot Inlets}

Figure 4 shows the profiles of the mean (top) and rms (bottom) streamwise velocity in the cold branch at $x=-3.1$ (left) and in the hot inlet branch at $z=2.14286$ (i.e., $\left.z^{*} / D_{H}=3\right)$ (right). The experimental data are plotted with symbols, while the blue solid line represents the Nek5000 simulation and red dashed line are the inlet profiles 

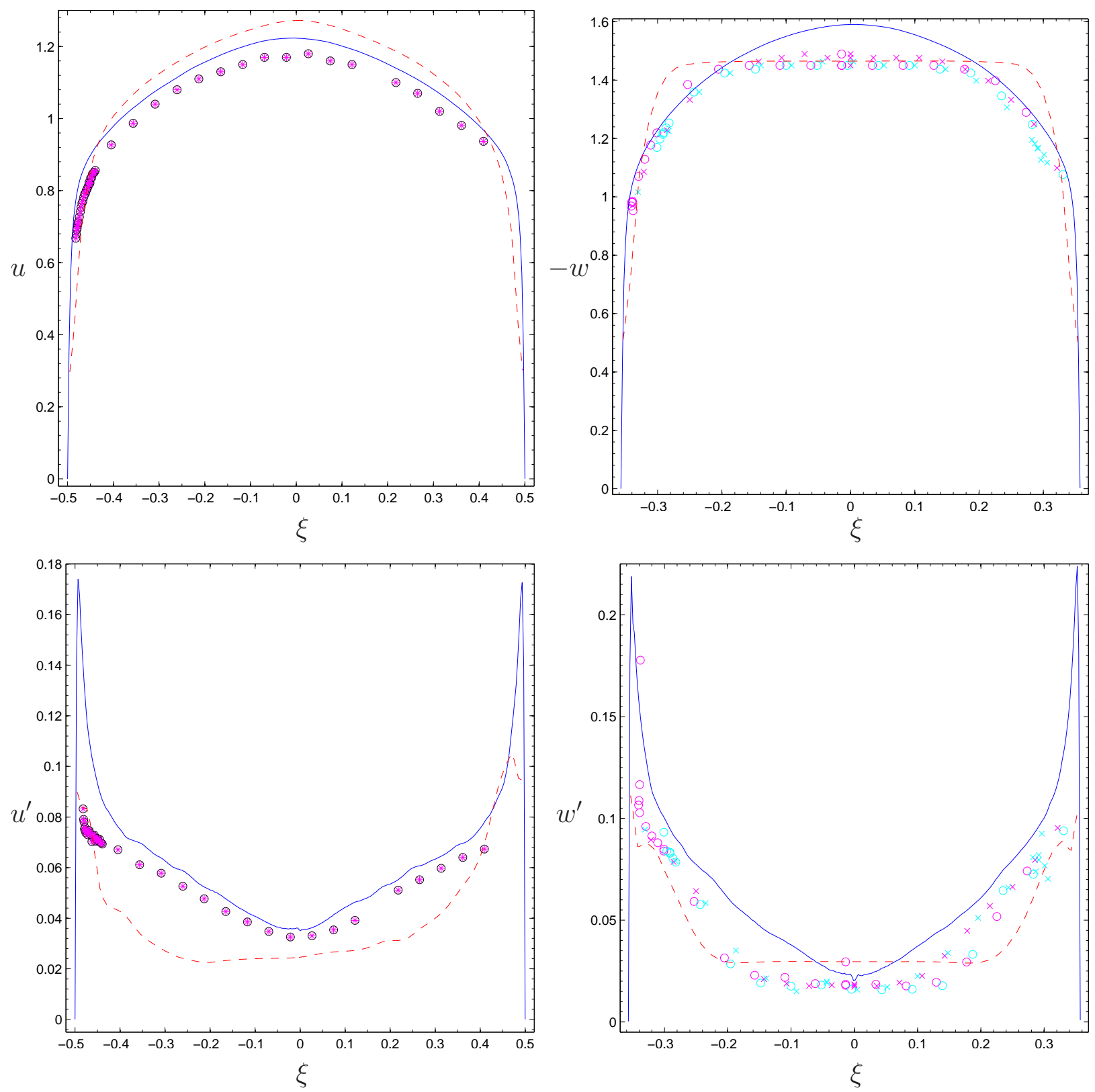

Figure 4. Mean (top) and rms (bottom) velocity profiles in the cold inlet branch (left) and in the hot inlet branch (right) vs a centerline coordinate $\xi$ for the experimental data (symbols) and simulation results with Nek5000 (blue solid line) and with CABARET (red dashed line). 

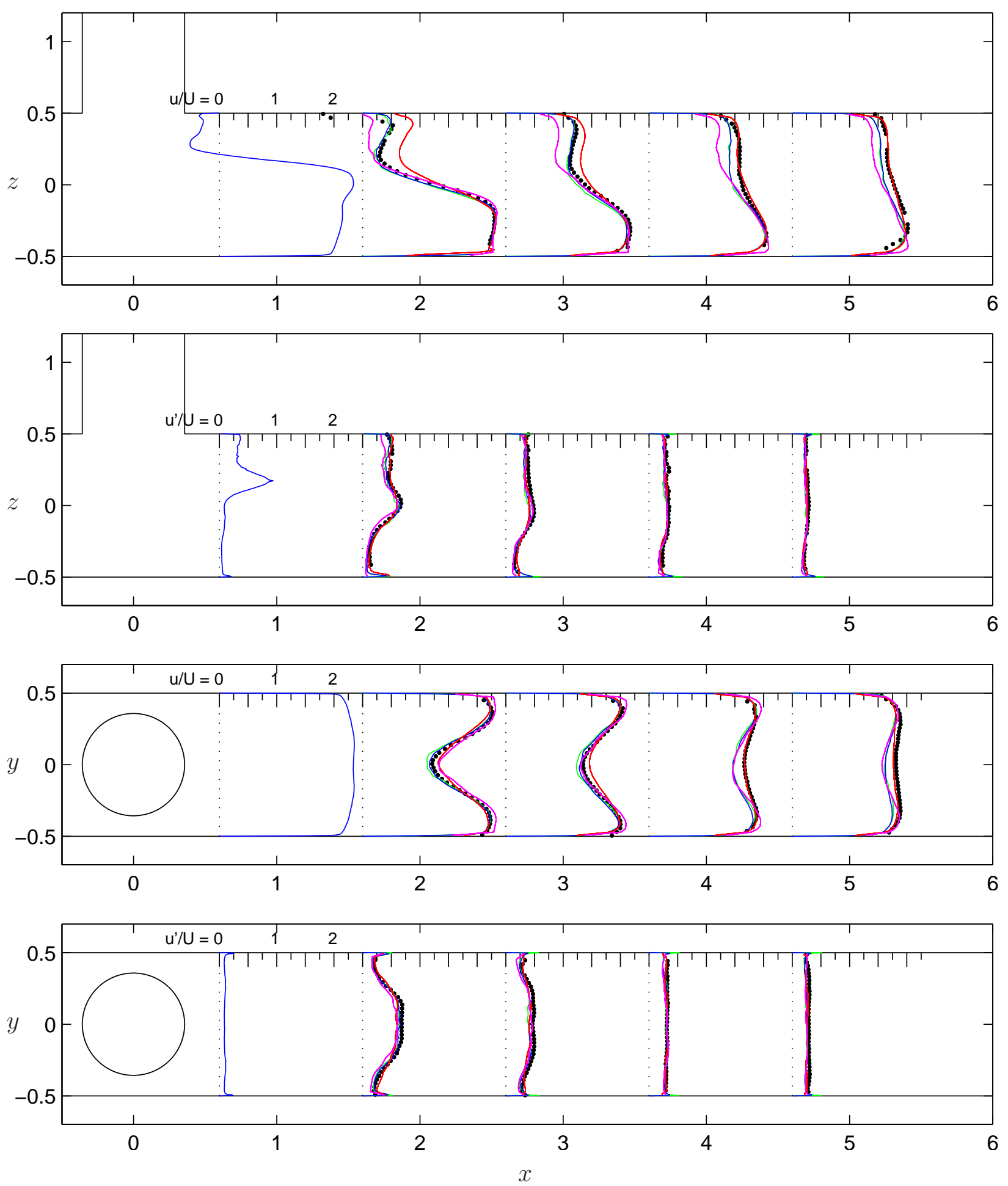

Figure 5. T-junction experimental data (.), CABARET (red), and Conv3D (magenta) results, and Nek5000 simulation for the benchmark submission results (green) and for the calculation with a longer time integration (blue line). From top to bottom: vertical profiles of axial mean and rms velocity, and horizontal profiles of axial mean and rms velocity. 
from the CABARET simulation. Note that the streamwise component of velocity for cold inlet coincides with the $x$-direction, while the hot flow in the hot inlet is in the direction opposite the $z$ coordinate; hence, $-w$ is plotted for the hot inlet. Also note that experimental data is plotted in the same style as in [22, Figures 5 and 6 or A5 and A8].

The nondimensional experimental data for the cold inlet does not integrate to unity, indicating a discrepancy between the reported flow rate and the LDA measurements of approximately $6 \%$. On the contrary, using the trapezoidal rule, the integrals of Nek5000 profiles for the cold and hot inlets are equal to 1.0027 and 1.3080, respectively, once averaged over and normalized by the corresponding inlet cross-sections. The same integration procedure for CABARET profiles in Figure 4 gives the normalized inlet flow rates equal to 1.014 and 1.330 for the cold and hot inlets, respectively. These values are in a good agreement with the nondimensional values for inlet velocities $U=1.000$ and $U_{H}=1.307$ given in Table 2. Note that the normalized inlet mass flow rates for Nek5000 were averaged over the $\xi=z$ line $(y=0)$ and $\xi=y$ line $(z=0)$ for the cold inlet and over the $\xi=x$ line $(y=0)$ and $\xi=y$ line $(x=0)$ for the hot inlet (Figure 4 ).

However, a comparison of the shape of the inlet simulation profiles with the experimental data reveals a few differences. The shape of the hot inlet profile for the mean and rms velocity for Nek5000 simulation is not as flat as the experimental or CABARET profiles. This difference can be attributed to a particular modeling of the non-fully-developed flow with the recycling technique described in Section 3. Note that the same technique works nearly perfectly for the cold inlet, where the profile of the mean velocity for Nek5000 is in excellent agreement with the experimental data points after multiplication by 0.94 factor to account for the mass conservation uncertainty of $6 \%$. Similarly, the cold inlet rms velocity for Nek5000 data shows good agreement, diverging from the experimental data points only in the near-wall region, which can be explained by the lower Reynolds number of the simulation, $R e=4 \times 10^{5}$ (cf. Table 2). These results show that further investigation is needed into the effects of the non-fully-developed flow in the hot inlet for Nek5000 simulation.

On the contrary, the CABARET simulation models the flat profile at the hot inlet well (Figure 4, right). However, the cold inlet profiles deviate substantially from the experimental data (Figure 4, left).

\subsubsection{Downstream of the T-Junction}

We next look at the axial mean $u$ and rms $u^{\prime}$ velocity downstream of the T-junction. Figure 5 shows a "bird-eye" view of the mean $(u)$ and $\mathrm{rms}\left(u^{\prime}\right)$ velocity profiles downstream of the T-junction at $x=0.6,1.6,2.6,3.6$, and 4.6. Here the experimental data (black symbols) are contrasted with numerical simulations with CABARET (red), Conv3D (magenta), and Nek5000 for the benchmark submission results (green) and for longer time integration/averaging (blue). Note the T-junction geometry outline and the equal unit scale for the mean and rms axial velocity equal to the velocity scale, namely, the axial mean velocity in the cold inlet branch $U=1.000$ (Table 2). For reference, we provide a detailed comparison of numerical simulations with experimental data points of the mean and rms axial velocity for each cross-section separately (Figures 6-13). Each figure shows the results of numerical simulations with Nek5000 (blue), CABARET (red), and Conv3D (pink) against the experimental data points (symbols). The vertical profiles of the mean 

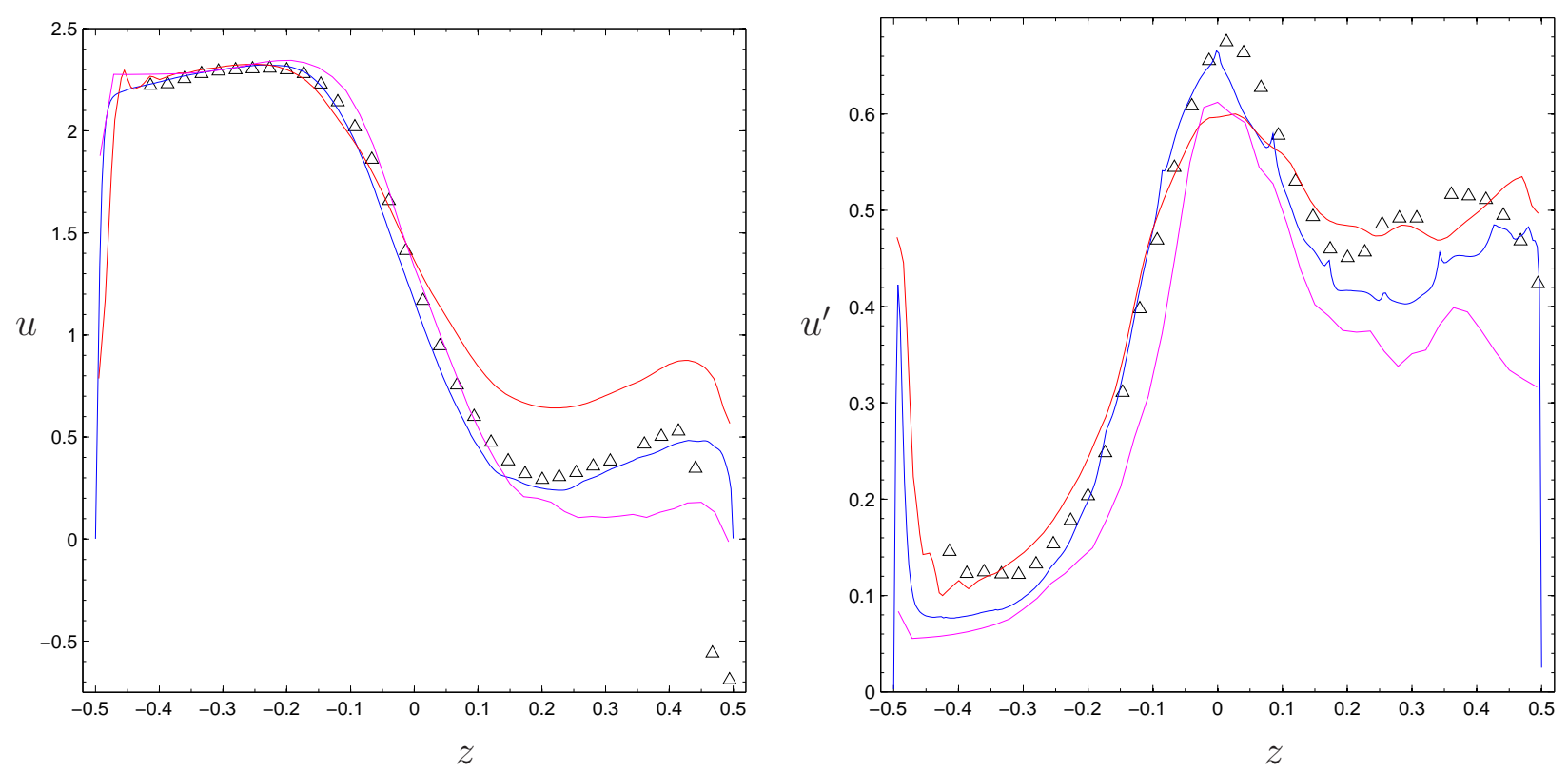

Figure 6. Axial mean velocity (left) and rms (right) vertical profiles at $x=1.6$ for experimental data (triangles) and simulation with Nek5000 (blue), CABARET (red), and Conv3D (magenta line).

$u$ (left) and rms $u^{\prime}$ (right) axial velocity are shown in Figures $6-9$ at $x=1.6 \ldots 4.6$, correspondingly, while the horizontal profiles are plotted similarly in Figures 10-13. Detailed comparison with Nek5000 benchmark submission results is reported in Section 6.2 (see Figure 14).

Overall agreement of simulation results for the three codes with the experimential data is good for the both mean and rms axial velocity. The Nek5000 profiles (blue lines) of the rms velocity match experimental data points well (Figures 5 and 6-13, right). Moreover, the agreement between the simulation and experiment for the mean velocity (left figures) is best at $x=2.6$ (Figures 7 and 11) and at $x=1.6$ (Figures 6 and 10) apart of two near-wall data points close to at $z=0.5$ (Figure 6). This discrepancy is the focus of an investigation described in Section 6.3. The deviation of the mean velocity for Nek5000 results from experimental data further downstream at $x=3.6$ and 4.6 in Figures $5,8-9$ and $12-13$ can be attributed to the lower Reynolds number used in the simulation because of the time constraints and will be the subject of a separate, follow-up study.

On the contrary, CABARET simulation results (red lines) agree with experiment remarkably well further downstream, at $x=3.6$ (Figures 8 and 12) and $x=4.6$ (Figures 9 and 13), with the notable exception of the best match of rms data with experimental points in the recirculation region at $x=1.6$ and $z>0$ (Figure 6). However, close to the T-junction, at $\mathrm{x}=1.6$ and 2.6, the CABARET profiles of the mean axial velocity deviate from experimental points at $z>0$ (Figures 6-7) and near the centerline $y=0$ (Figures 10-11). Similar to CABARET profiles, the Conv3D results (pink lines) show the 

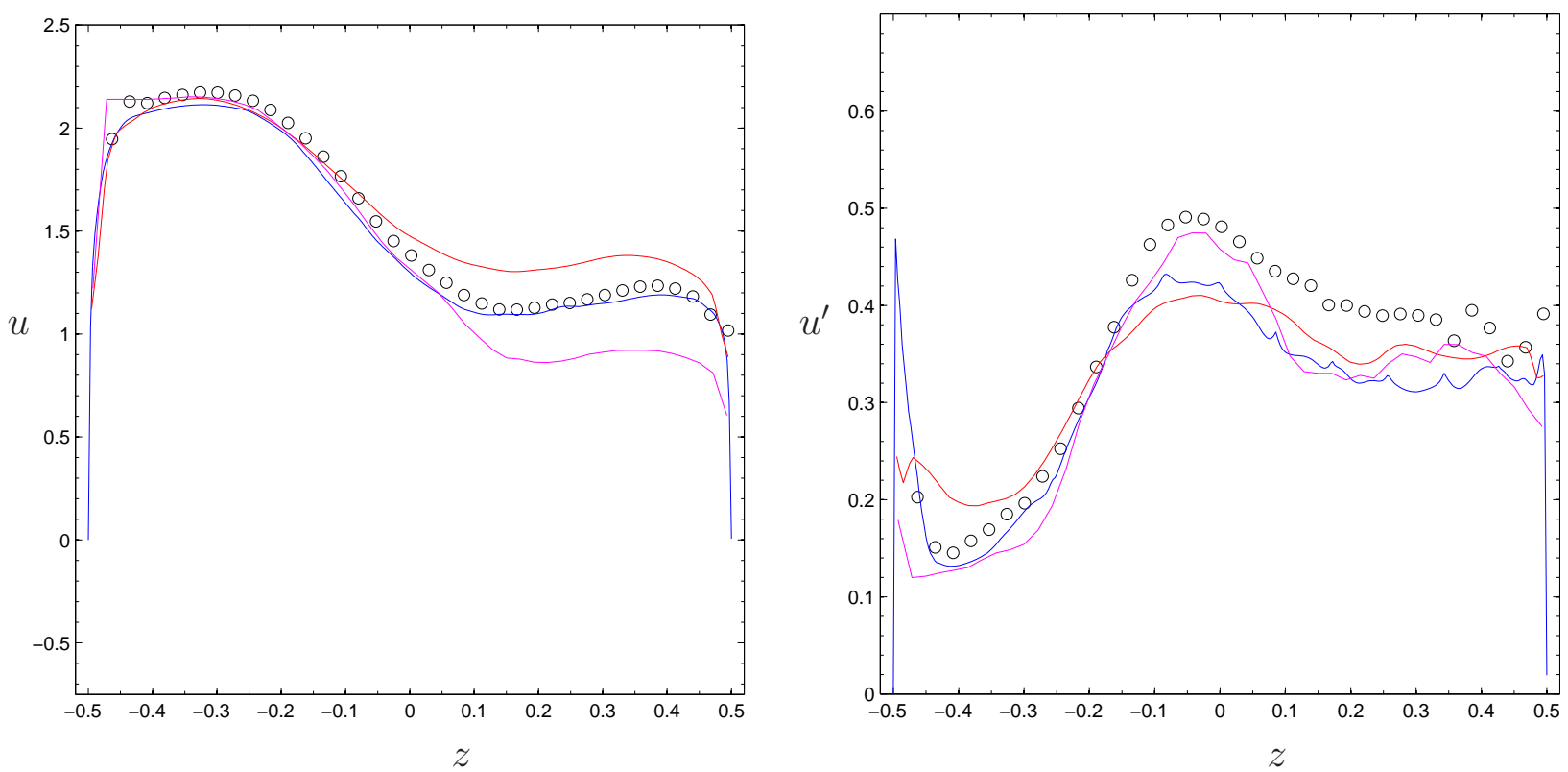

Figure 7. Axial mean velocity (left) and rms (right) vertical profiles at $x=2.6$ for experimental data (circles) and simulation with Nek5000 (blue), CABARET (red), and Conv3D (magenta line).

most deviation from experimental data for the mean axial velocity at $z>0$ (Figures 69 ) and near the centerline $y=0$ (Figures 10-13). However, the agreement of Conv3D simulation with experiment is best at $x=4.6$ and $0.4<|y|<0.5$ (Figure 13).

\subsection{Sensitivity Study}

To study the effects of increasing resolution and time averaging interval, we performed an additional set of simulations with Nek5000, CABARET, and Conv3D.

In addition to the benchmark submission results with $N=7$ (i.e., $n \approx E N^{3} \approx 2.1 \times 10^{7}$ points), Nek5000 runs were conducted with $N=5\left(n \approx 7.7 \times 10^{6}\right.$ points $)$. These runs started with the $N=7$ results and were run and averaged over about 110 convective time units (i.e., about 26 seconds). The timestep size was $\Delta t=5 \times 10^{-4}$ and required 5.6 seconds per timestep on 410 nodes of the SciCortex. Each node of the SciCortex has 6 cores at $550 \mathrm{MHz}$, but the simulations were run with the nodes undersubscribed to use only 5 cores each, which yielded a 10x performance gain over the fully-subscribed case.

The following figures highlight the mesh sensitivity of velocity profiles extracted from results of numerical simulations with Nek5000 (Figure 14), CABARET (Figures 15-18), and Conv3D (Figures 19-22). Each figure compares the vertical and horizontal profiles of the mean axial velocity $u$ and its rms $u^{\prime}$ for different meshes with the experimental data (symbols).

For the Nek5000 simulations, Figure 14 shows the vertical (left) and horizontal (right) profiles of the mean (top) and rms (bottom) axial velocity profiles at $x=0.6$ (magenta), 1.6 (black), 2.6 (blue), 3.6 (green), and 4.6 (red) with $N=5$ (dashed) and $N=7$ 

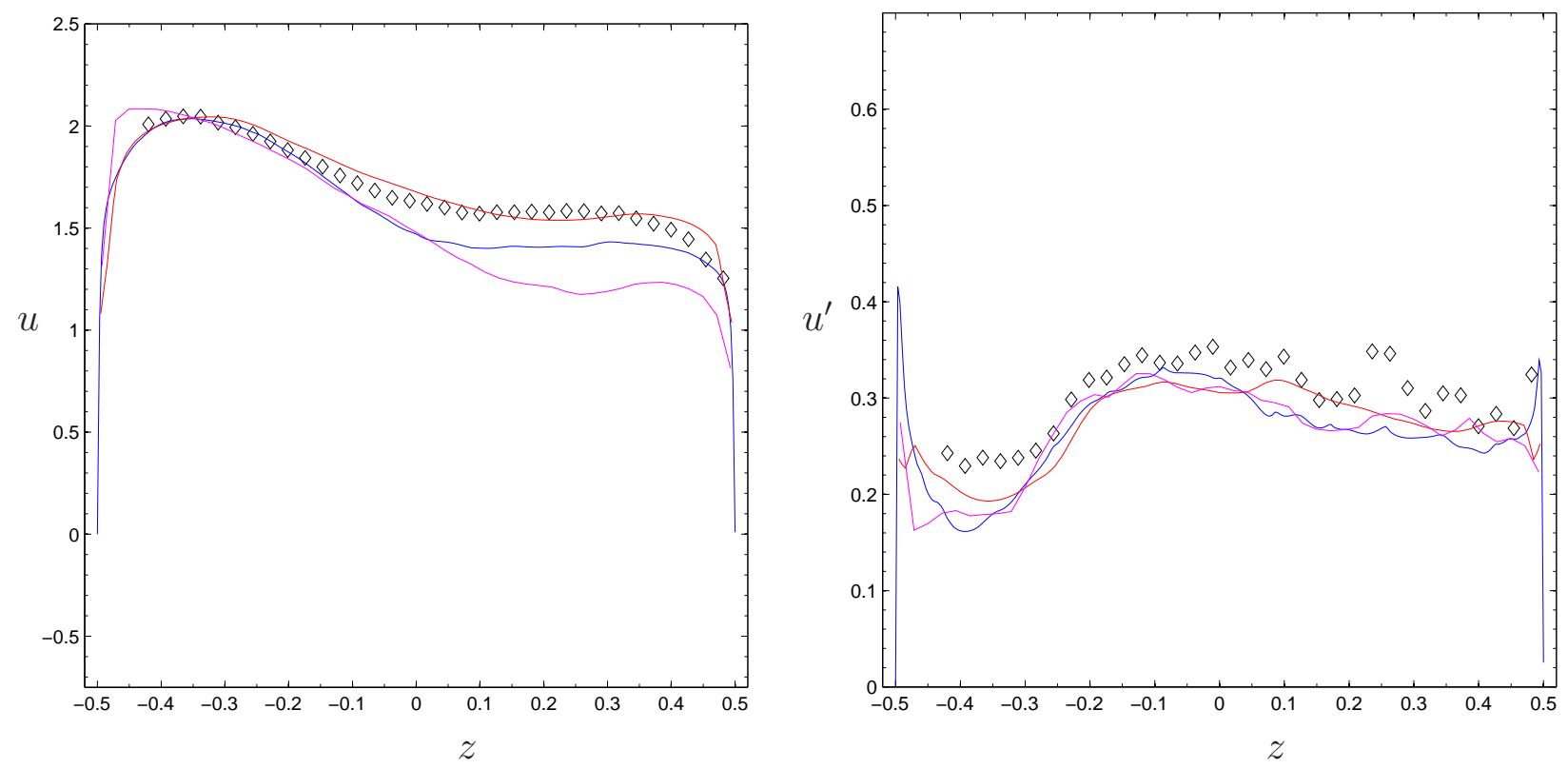

Figure 8. Axial mean velocity (left) and rms (right) vertical profiles at $x=3.6$ for experimental data (diamonds) and simulation with Nek5000 (blue), CABARET (red), and Conv3D (magenta line).

(solid). The benchmark submission results (i.e., with $N=7$ and shorter time average) plotted with dash-dotted line at $x=1.6 \ldots 4.6$ are in the excellent agreement with the longer time average run (solid). All profiles agree well with the experimental data points (symbols) especially considering the fact that the Reynolds number of these simulations, $R e=4 \times 10^{5}$, is twice lower than that of the experiment (see Table 2). In general, the profiles from the coarse mesh simulation (i.e., $N=5$, dashed) are close to the solution for the finer mesh (solid and dashed-dotted lines). Note the excellent agreement between the profiles for $N=5$ and $N=7$ at $x=0.6$, where the strength of the reversed flow is close to its peak (Figure 23). The largest deviation in the profiles (up to about 0.2) is observed at $x=1.6$ and $0.1<z<0.25$; thus a further study is warranted with even finer mesh, say, $N=8$ or $N=9$.

For the CABARET simulations, the vertical profiles of $u$ (left) and $u^{\prime}$ are plotted in Figures $15-16$ at $x=1.6 \ldots 4.6$, and the horizontal profiles at the same $x$ locations are shown in Figures 17-18. These figures show results from CABARET simulations on a coarser mesh with 0.5 million points averaged over a half time interval (red dotted) and the full time interval (blue dash-dotted) and on a finer mesh with 4 million points averaged over a half time interval (green dashed) and the full time interval (solid black line). Note the excellent convergence for the mean axial velocity profiles at $x=3.6$ and $x=4.6$ (Figures 16 and 18, left).

For the Conv3D simulations, the vertical profiles of $u$ (left) and $u^{\prime}$ are plotted in Figures $19-20$ at $x=1.6 \ldots 4.6$, and the horizontal profiles at the same $x$ locations are 

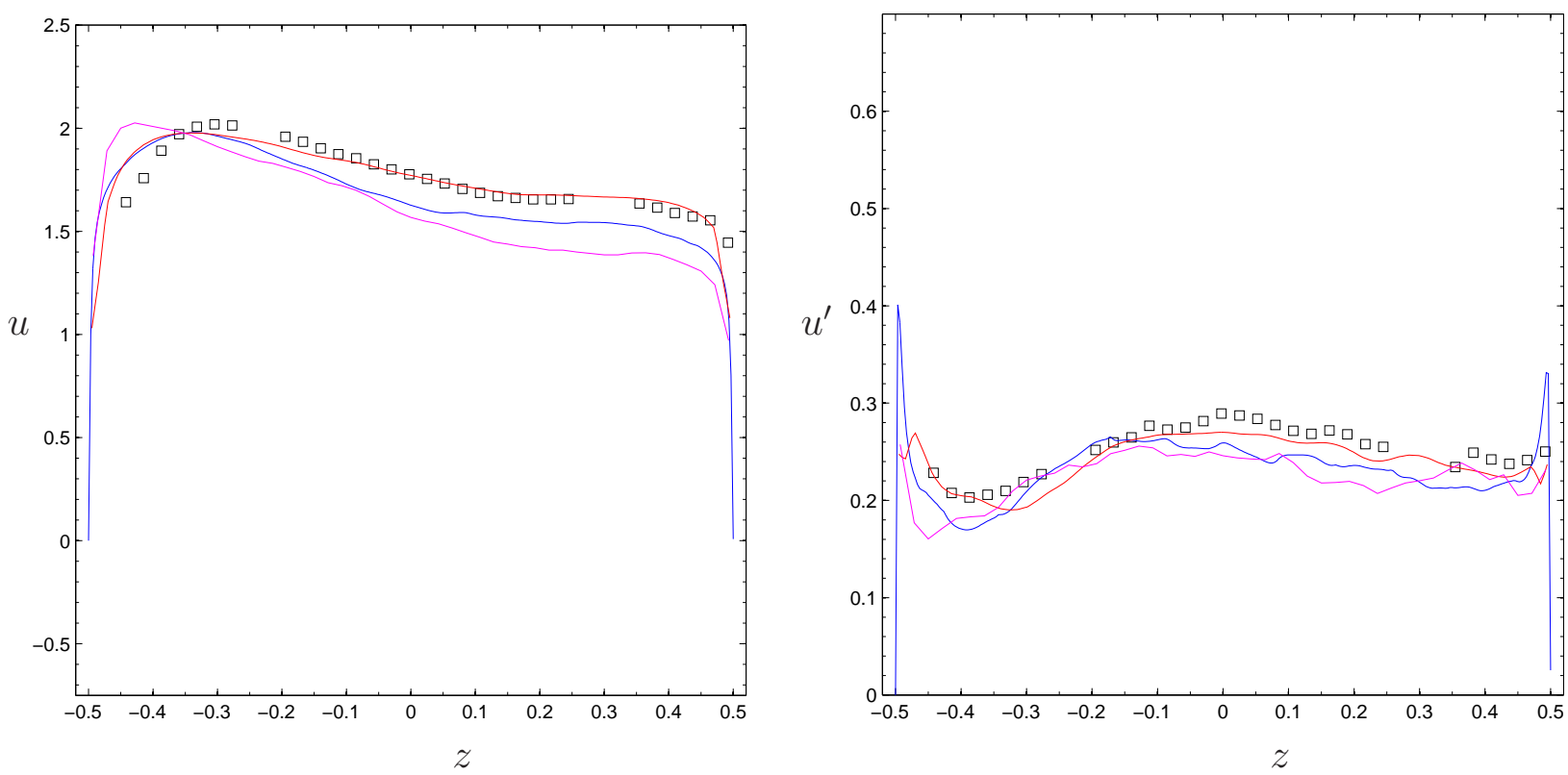

Figure 9. Axial mean velocity (left) and rms (right) vertical profiles at $x=4.6$ for experimental data (squares) and simulation with Nek5000 (blue), CABARET (red), and Conv3D (magenta line).

shown in Figures 21-22. These figures show results from Conv3D simulations on a 40 million node uniform (black solid) and nonuniform (blue dash-dotted) mesh, on a 12 million node uniform mesh (green dashed), and on a 3 million node nonuniform mesh (red dotted line). Note the good convergence for the mean axial velocity profiles (left figures) at $x=1.6 \ldots 3.6$ and $z=<0$ (Figures 19 and 20, top).

\subsection{Study of Reversed Flow Region}

To address the discrepancy between experimental data and simulations near the upper wall at $x=1.6$, we have also undertaken a series of Nek5000 simulations to investigate the sensitivity of the reattachment of the recirculation region downstream of the T-junction.

Summarizing the effects of Reynolds number, grid resolution, and time averaging on the reversed flow region, Figure 23 shows time-averaged velocity profiles near the upper wall at $z=0.005$ and $y=0$ for Nek5000 simulations at $R e=9 \times 10^{4}$ with $N=11$ (red), at $R e=6 \times 10^{4}$ with $N=9$ (cyan) and at $R e=4 \times 10^{4}$ with $N=9$ (magenta), $N=5$ (black) and $N=7$ for benchmark submission results (green) and longer time averaging (blue). Note the dotted lines that correspond to experimental profile measurements and $u=0$ value.

Based on this study, we conclude that the reattachment region is insensitive mainly to an increase in Reynolds number and grid resolution. Moreover, results at $R e=4 \times 10^{4}$ indicate that the recirculation region is not sensitive to the averaging/integration time interval. Ideally, one should conduct a further investigation at higher Reynolds number with longer integration/averaging time to resolve the discrepancy between the simulations 

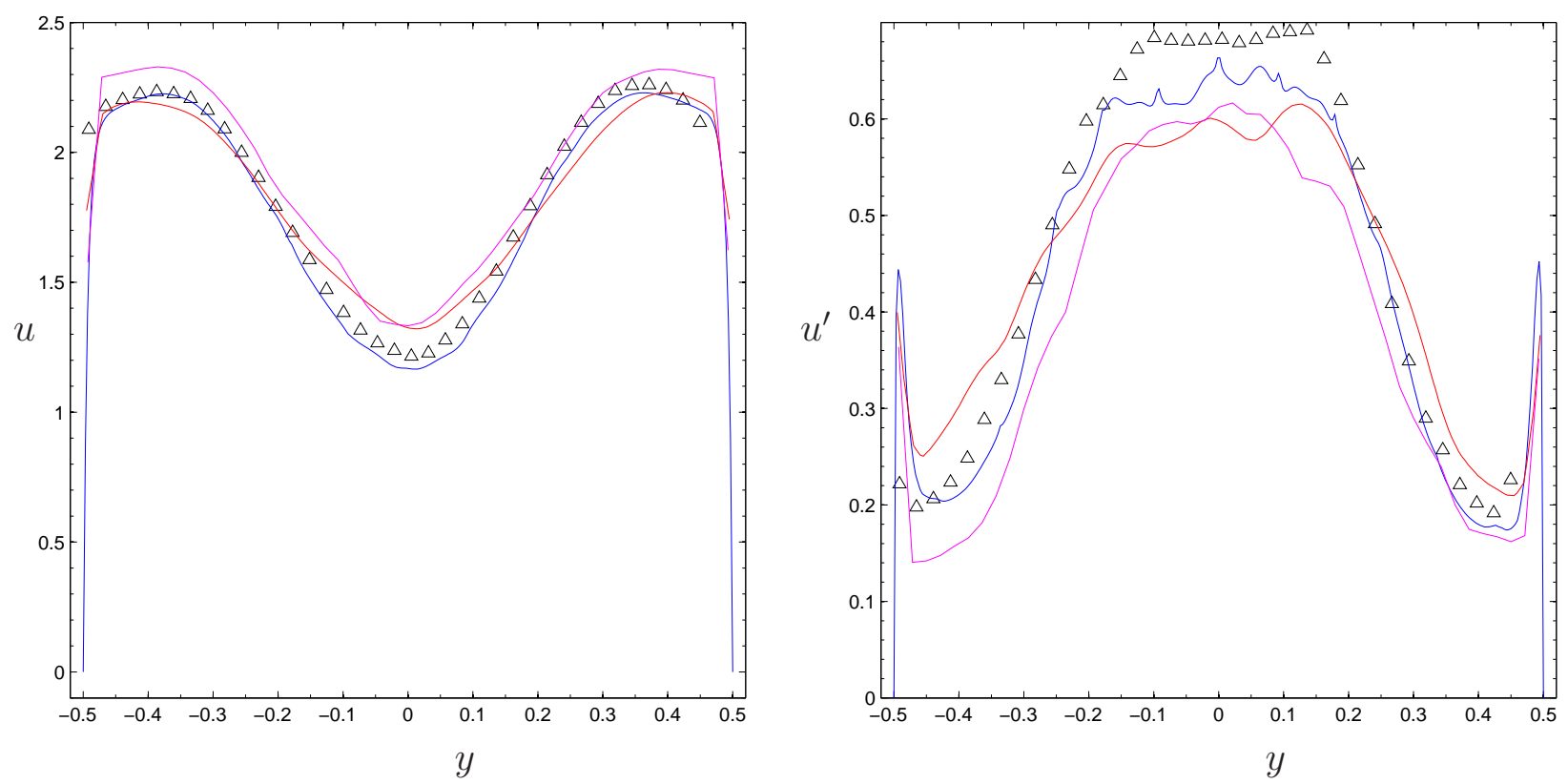

Figure 10. Axial mean velocity (left) and rms (right) horizontal profiles at $x=1.6$ for experimental data (triangles) and simulation with Nek5000 (blue), CABARET (red), and Conv3D (magenta line).

and experimental data points near the upper wall at $x=1.6$. For now, we quote that in the experimental measurements of velocity, "... the focus ... is not the near-wall region" $[22, \mathrm{p} .15]$.

\section{Conclusions and Future Work}

We have compared and contrasted three simulation approaches to modeling unsteady thermal transport on a problem relevant to the design of nuclear power plants. Specifically, we investigate a finite-difference ILES and finite-volume LES methods on fully staggered grids and an LES SEM method applied to a problem of simulation of the OECD/NEA benchmark of thermal stripping in T-junction.

The agreement of CABARET, Conv3D and Nek5000 with the experimental data is good, including the blind benchmark submission results for Nek5000. Comparison with experiment is even more favorable in light of two considerations. First, the mass flow rate uncertainty in the experimental inlet profile reported here is up to $6 \%$. Second, most of the Nek5000 simulations were conducted at a Reynolds number which is a half that of the experimental value, because of time constraints. Nevertheless, the Nek5000 profiles match the experimental data exceptionally well closer to the T-junction, while the CABARET results agree remarkably well with experiment farther downstream. The only deviation of Nek5000 profiles from experimental data closer to the T-junction is observed near the upper wall at $x=1.6$. This deviation was the subject of an additional sensitivity study from which we concluded that the reattachment region was insensitive mainly to changes 

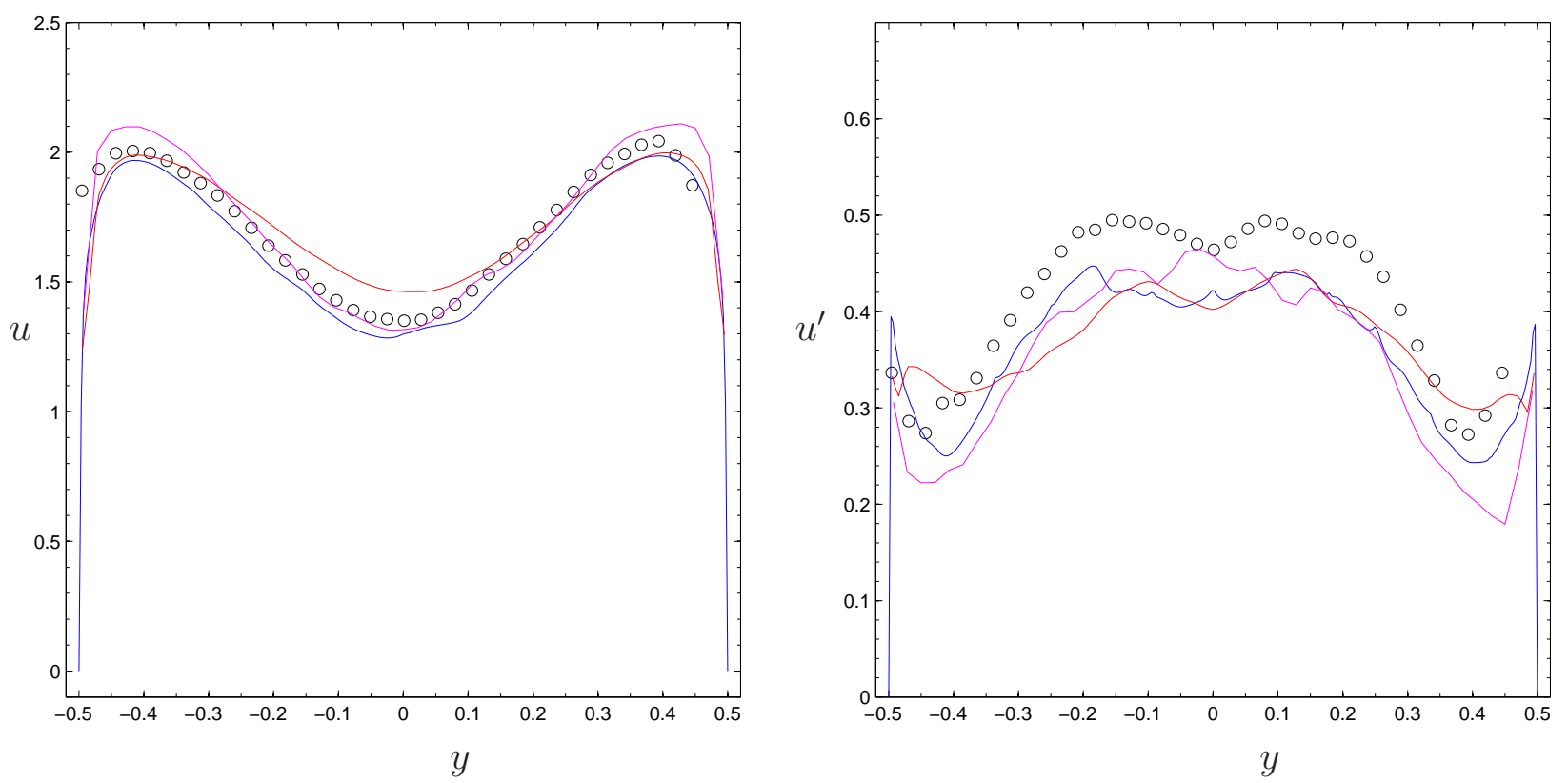

Figure 11. Axial mean velocity (left) and rms (right) horizontal profiles at $x=2.6$ for experimental data (circles) and simulation with Nek5000 (blue), CABARET (red), and Conv3D (magenta line).

in grid resolution, the Reynolds number and time integration interval. The results from a similar sensitivity study for velocity profiles from all three codes were also reported.

A follow-up study will include analysis of spectra for velocity and temperature from the three codes and will be a basis for a supplementary report [7]. Further Nek5000 computations at higher Reynolds number are desirable, including more modeling of the hot inlet profile to capture its flatness observed in experiment and achieved in CABARET simulations.

\section{Acknowledgments}

This work was supported by the National Institutes of Health, RO1 Research Project Grant (2RO1HL55296-04A2), by Whitaker Foundation Grant (RG-01-0198), and by Office of Advanced Scientific Computing Research, Office of Science, U.S. Department of Energy, under Contract DE-AC02-06CH11357. The work was completed as part of the SHARP reactor performance and safety code suite development project, which is funded under the auspices of the Nuclear Energy Advanced Modeling and Simulation (NEAMS) program of the U.S. Department of Energy Office of Nuclear Energy.

One of the authors would like to thank Gary Leaf, Elia Merzari, and Dave Pointer for helpful discussions, and his wife Alla for her patience during his work on this report. 

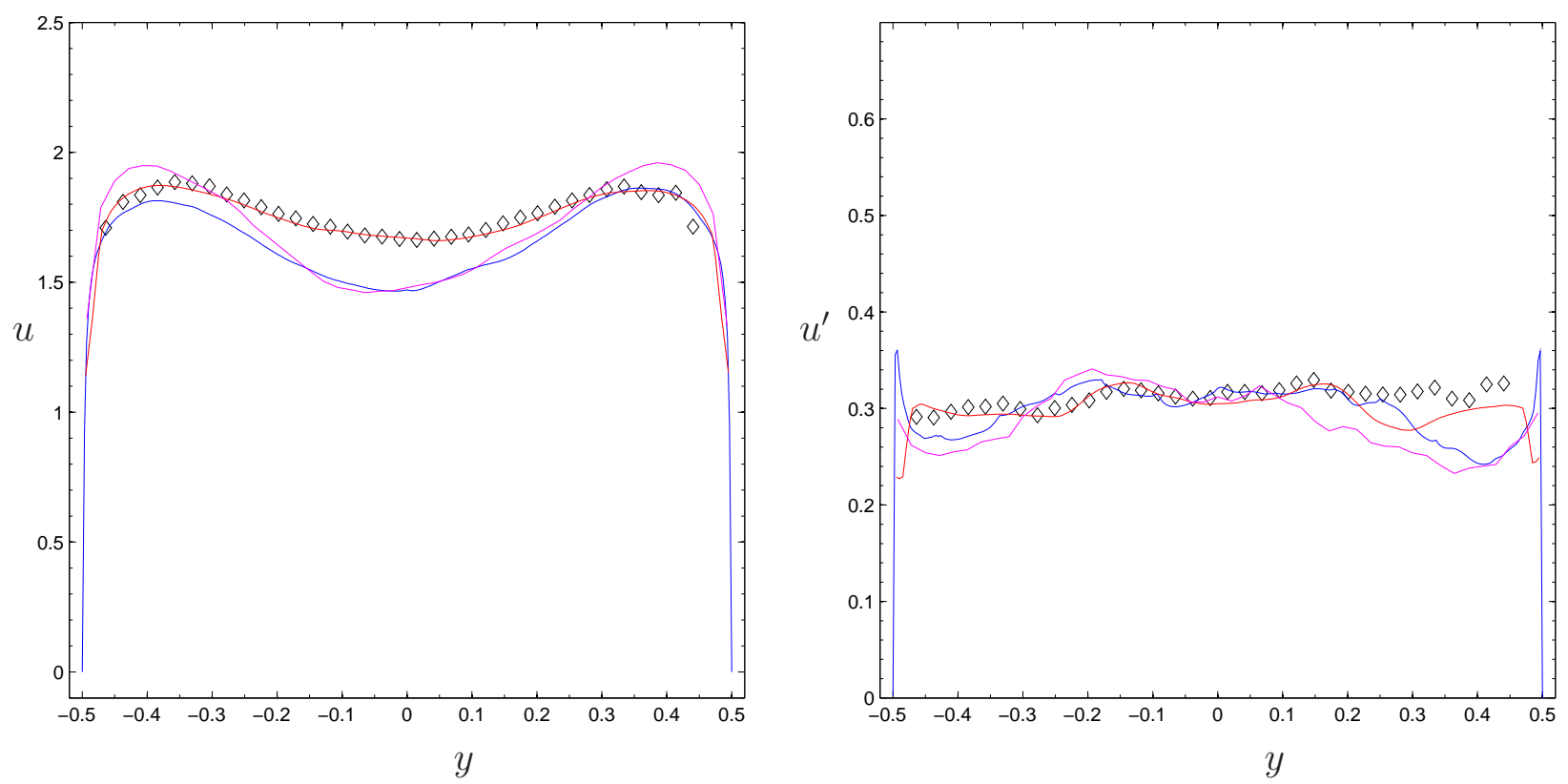

Figure 12. Axial mean velocity (left) and rms (right) horizontal profiles at $x=3.6$ for experimental data (diamonds) and simulation with Nek5000 (blue), CABARET (red), and Conv3D (magenta line).

\section{REFERENCES}

1. L.A. Bolshov, V.V. Chudanov, V.F. Strizhov, S.V. Alekseenko, V.G. Meledin, and N.A. Pribaturin, Best estimate methodology for modeling bubble flows, 14th International Conference on Nuclear Engineering (ICONE14) CD-ROM (Miami, Florida), no. ICONE-89296, 2006.

2. V.V. Chudanov, The integrated approach to a solution of CFD problems in complicated areas, 1999, (in Russian), pp. 126-135.

3. V.V. Chudanov, A.E. Aksenova, and V.A. Pervichko, 3D unified CFD approach to thermalhydraulic problems in safety analysis, IAEA Technical Meeting on Use of Computational Fluid Dynamics (CFD) Codes for Safety Analysis of Nuclear Reactor Systems, Including Containment CD-ROM (Pisa, Italy), no. IAEA-TECDOC-1379, 2003.

4. __ CFD to modelling molten core behaviour simultaneously with chemical phenomena, 11th International Topical Meeting on Nuclear Reactor Thermal-Hydraulics (NURETH-11) CD-ROM (Avignon, France), no. 048, 2005.

5. _ Methods of direct numerical simulation of turbulence in thermalhydraulic's problems of fuel assembly, Izvestiya Rossiiskoi Akademii Nauk. Seriya Energetica 6 (2007), 47-57.

6. M.O. Deville, P.F. Fischer, and E.H. Mund, High-order methods for incompressible fluid flow, Cambridge University Press, Cambridge, 2002.

7. A.V. Obabko et al., Tech. report, Argonne National Lab, 2011, in preparation. 

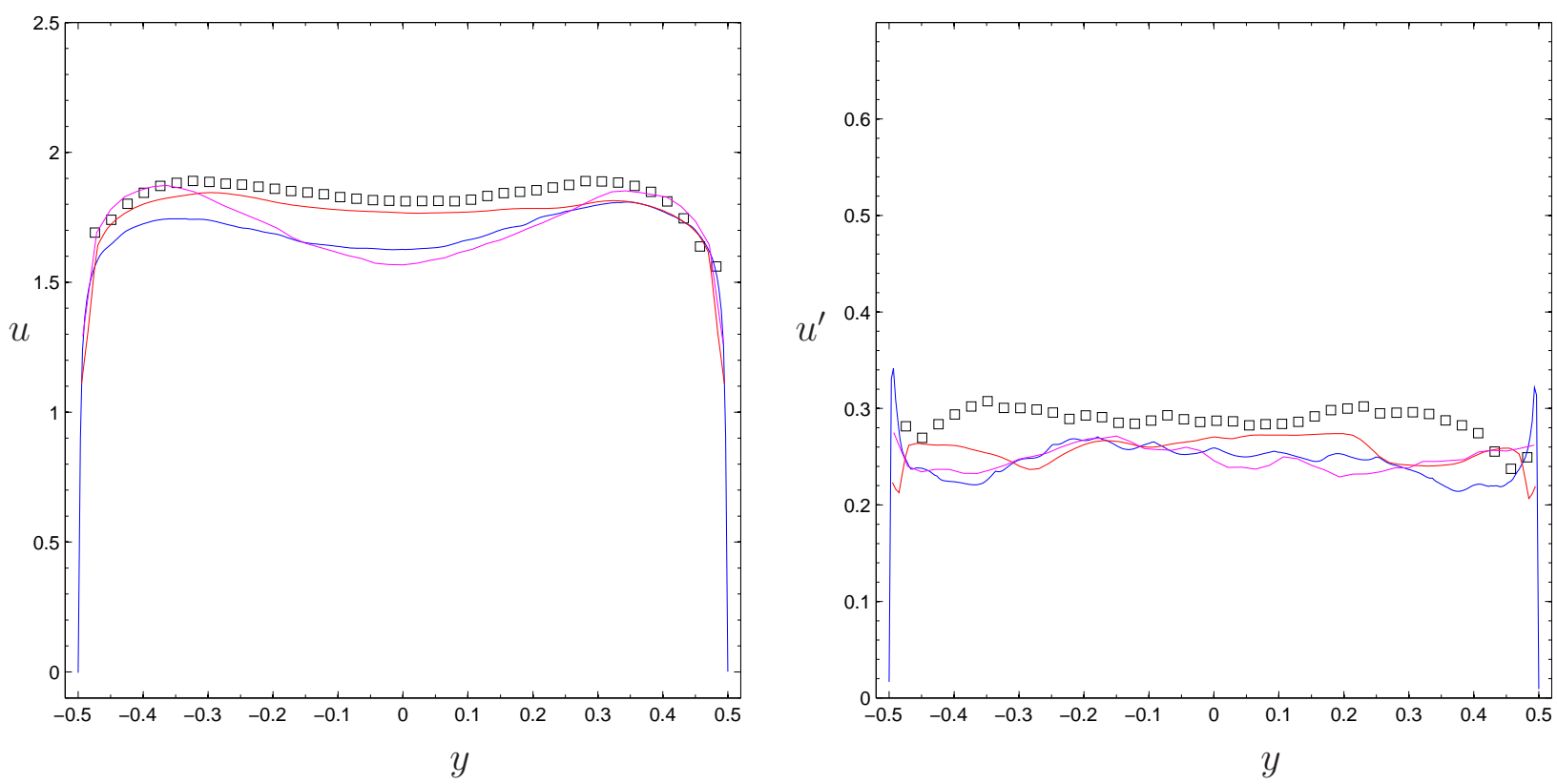

Figure 13. Axial mean velocity (left) and rms (right) horizontal profiles at $x=4.6$ for experimental data (squares) and simulation with Nek5000 (blue), CABARET (red), and Conv3D (magenta line).

8. Chudanov et al., Journal of Computing Mathematics and of Mathematical Physics 40 (2001), 900-907.

9. P.F. Fischer, An overlapping Schwarz method for spectral element solution of the incompressible Navier-Stokes equations, J. Comput. Phys. 133 (1997), 84-101.

10. P.F. Fischer and A.T. Patera, Parallel spectral element of the Stokes problem, J. Comput. Phys. 92 (1991), 380-421.

11. V.M. Goloviznin, Balanced characteristic method for systems of hyperbolic conservation laws, Doklady Mathematics 72 (2005), no. 1, 619-623.

12. V.M. Goloviznin, V.N. Semenov, I.A Korortkin, and S.A. Karabasov, A novel computational method for modelling stochastic advection in heterogeneous media, Transport in Porous Media 66 (2007), no. 3, 439-456.

13. F.F. Grinstein, L.G. Margolin, and W.J. Rider, Implicit large eddy simulation: Computing turbulent fluid dynamics, Cambridge University Press, 2007.

14. A. Iserles, Generalized leapfrog methods, IMA Journal of Numerical Analysis 6 (1986), no. 3, 381-392.

15. S. A. Karabasov and V. M. Goloviznin, Compact accurately boundary-adjusting highresolution technique for fluid dynamics, J. Comput. Phys. 228 (2009), 7426-7451.

16. S.A. Karabasov, P.S. Berloff, and V.M. Goloviznin, Cabaret in the ocean gyres, Ocean Modelling 30 (2009), no. 2-3, 155 - 168.

17. S.A. Karabasov and V.M. Goloviznin, Cabaret in the ocean gyres, New Efficient HighResolution Method for Non-Linear problems in Aeroacoustics 45 (2007), no. 12, 2861 
$-2871$.

18. Y. Maday and A.T. Patera, Spectral element methods for the Navier-Stokes equations, State-of-the-Art Surveys in Computational Mechanics (A.K. Noor and J.T. Oden, eds.), ASME, New York, 1989, pp. 71-143.

19. J. H. Mahaffy and B. L. Smith, Synthesis of benchmark results, Experimental Validation and Application of CFD and CMFD Codes to Nuclear Reactor Safety Issues (CFD4NRS-3) CD-ROM (Washington, DC), 2010.

20. A.T. Patera, A spectral element method for fluid dynamics : laminar flow in a channel expansion, J. Comput. Phys. 54 (1984), 468-488.

21. A.A. Samarskii and V.M. Goloviznin, Difference approximation of convective transport with spatial splitting of time derivative, Mathematical Modelling 10 (1998), no. 1, 86100.

22. B. L. Smith, J. H. Mahaffy, K. Angele, and J. Westin, Experiments and unsteady CFD-calculations of thermal mixing in a T-junction, Nuclear Engineering and Design (2012), to appear.

23. A.G. Tomboulides, M. Israeli, and G.E. Karniadakis, Efficient removal of boundarydivergence errors in time-splitting methods, J. Sci. Comput. 4 (1989), 291-308.

24. A.G. Tomboulides, J.C.Y. Lee, and S.A. Orszag, Numerical simulation of low Mach number reactive flows, Journal of Scientific Computing 12 (June 1997), 139-167.

25. A.G. Tomboulides and S.A. Orszag, A quasi-two-dimensional benchmark problem for low Mach number compressible codes, J. Comput. Phys. 146 (1998), 691-706.

26. J. Westin et al., Experiments and unsteady CFD-calculations of thermal mixing in a T-junction, Benchmarking of CFD Codes for Application to Nuclear Reactor Safety (CFD4NRS) CD-ROM (Garching, Munich, Germany), 2006. 

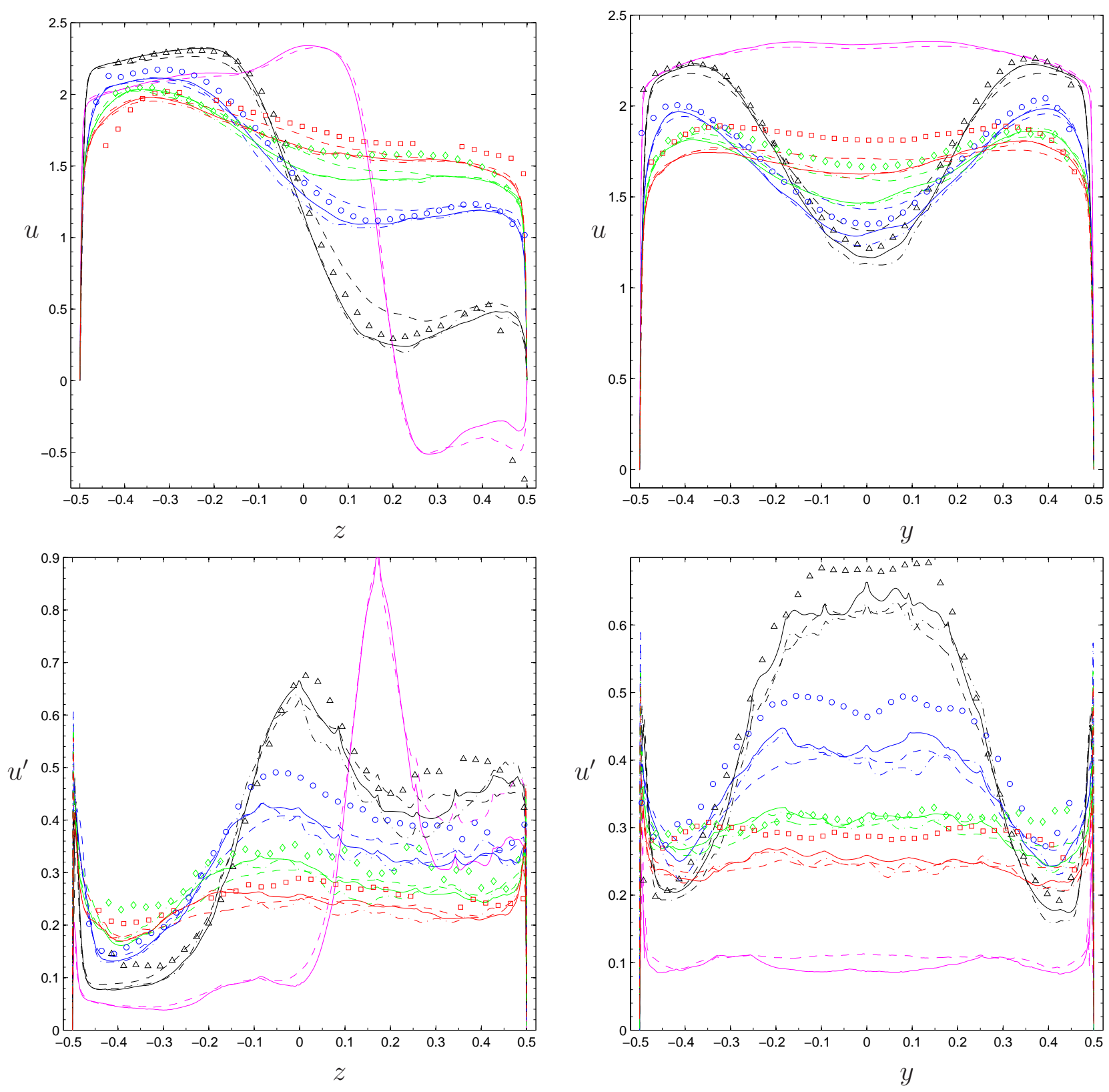

Figure 14. Vertical (left) and horizontal (right) profiles of axial mean velocity (top) and rms (bottom) profiles at $x=0.6$ (magenta), 1.6 (black), 2.6 (blue), 3.6 (green), and 4.6 (red) for Nek5000 simulation with $N=5$ (dashed) and $N=7$ (solid) and for Nek5000 benchmark submission results (dash-dot). 

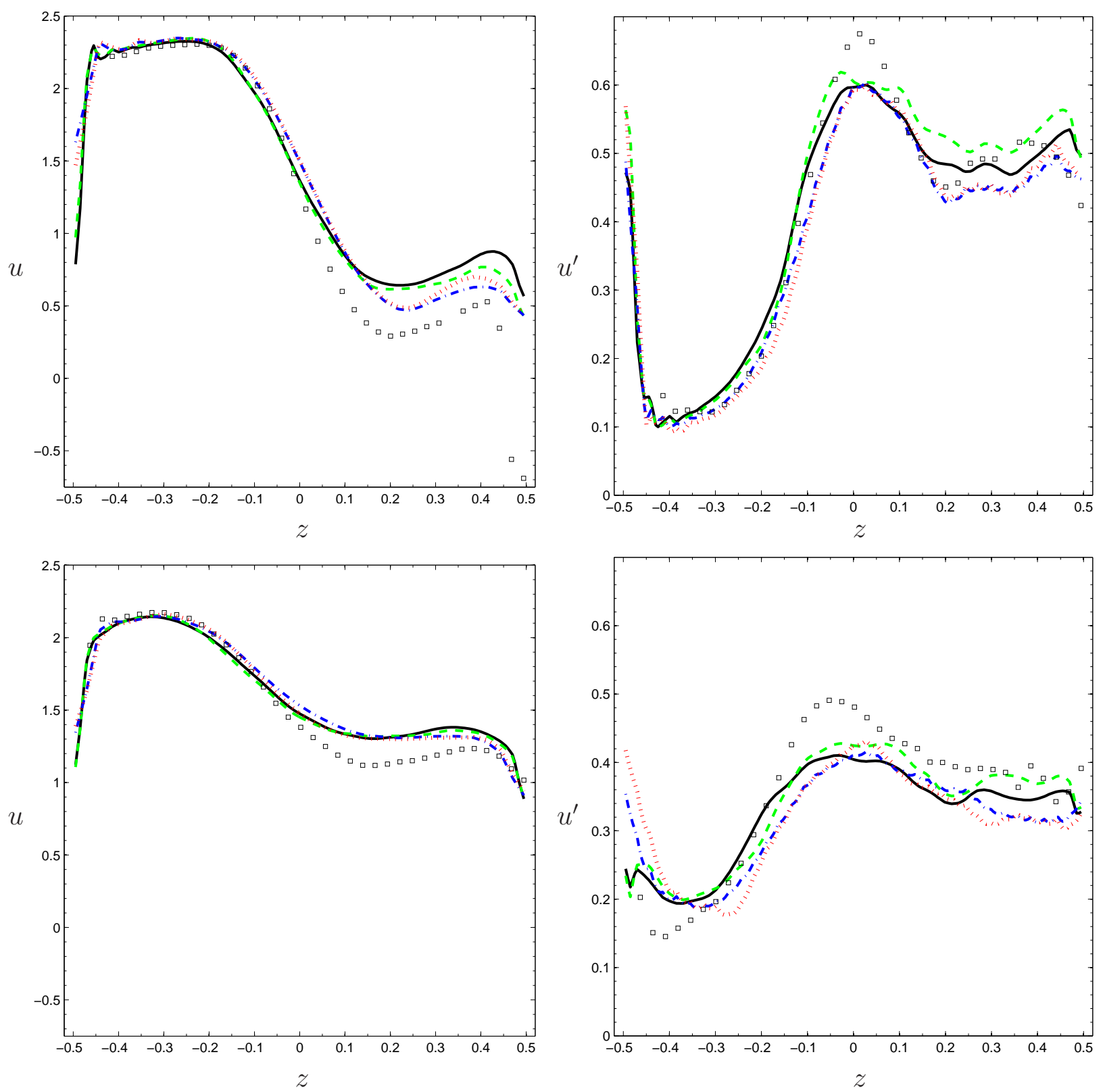

Figure 15. Vertical profiles of axial mean (left) and rms velocity (right) at $x=1.6$ (top) and $x=2.6$ (bottom) for the experimental data (symbols) and CABARET simulation on a coarser mesh with 0.5 million ponts averaged over a half (red dotted) and full (blue dash-dotted) interval, and on a finer mesh with 4 million points averaged over a half (green dashed) and full (solid black line) time interval. 

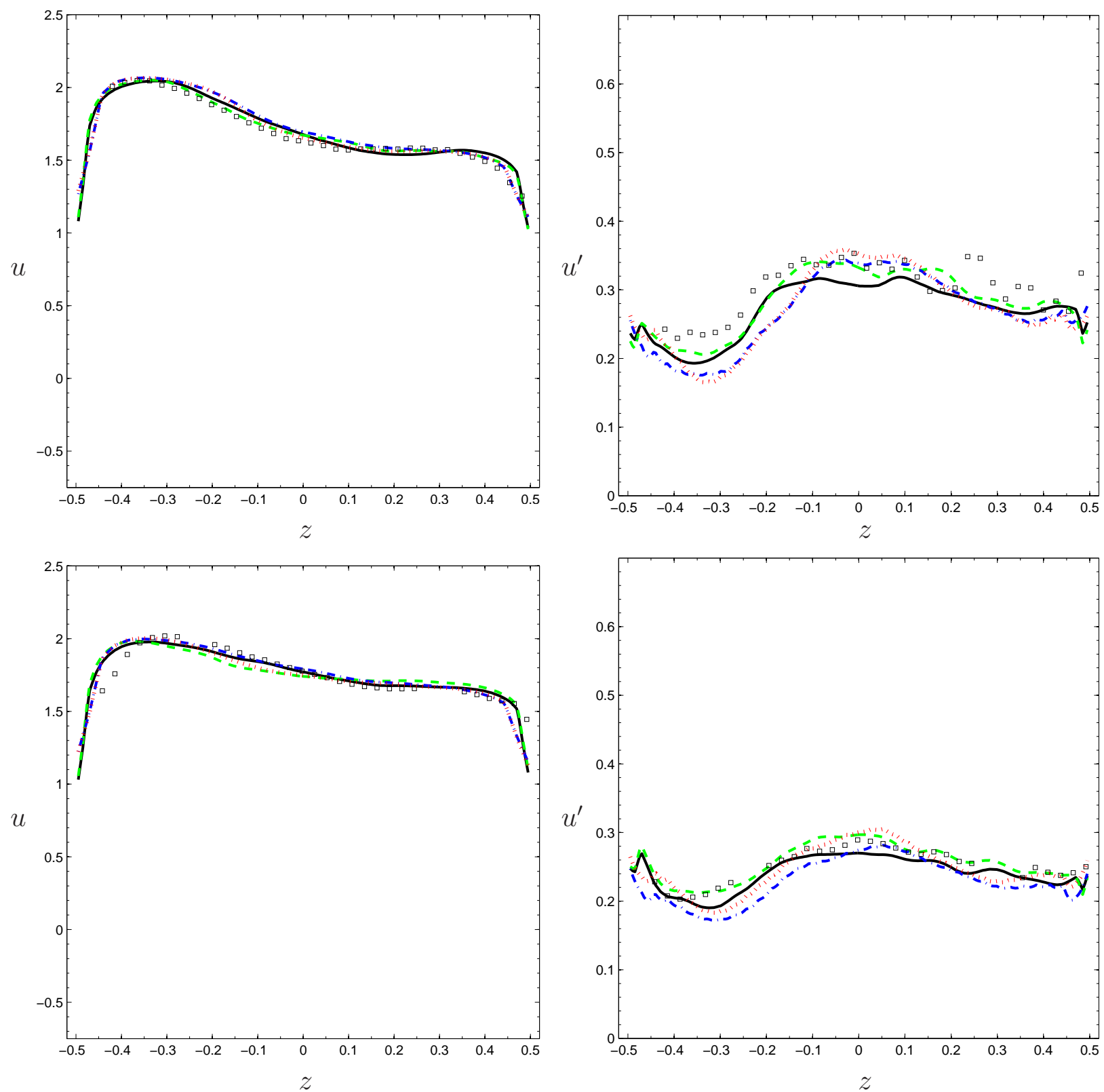

Figure 16. Vertical profiles of axial mean (left) and rms velocity (right) at $x=3.6$ (top) and $x=4.6$ (bottom) for the experimental data (symbols) and CABARET simulation on a coarser mesh with 0.5 million ponts averaged over a half (red dotted) and full (blue dash-dotted) interval, and on a finer mesh with 4 million points averaged over a half (green dashed) and full (solid black line) time interval. 

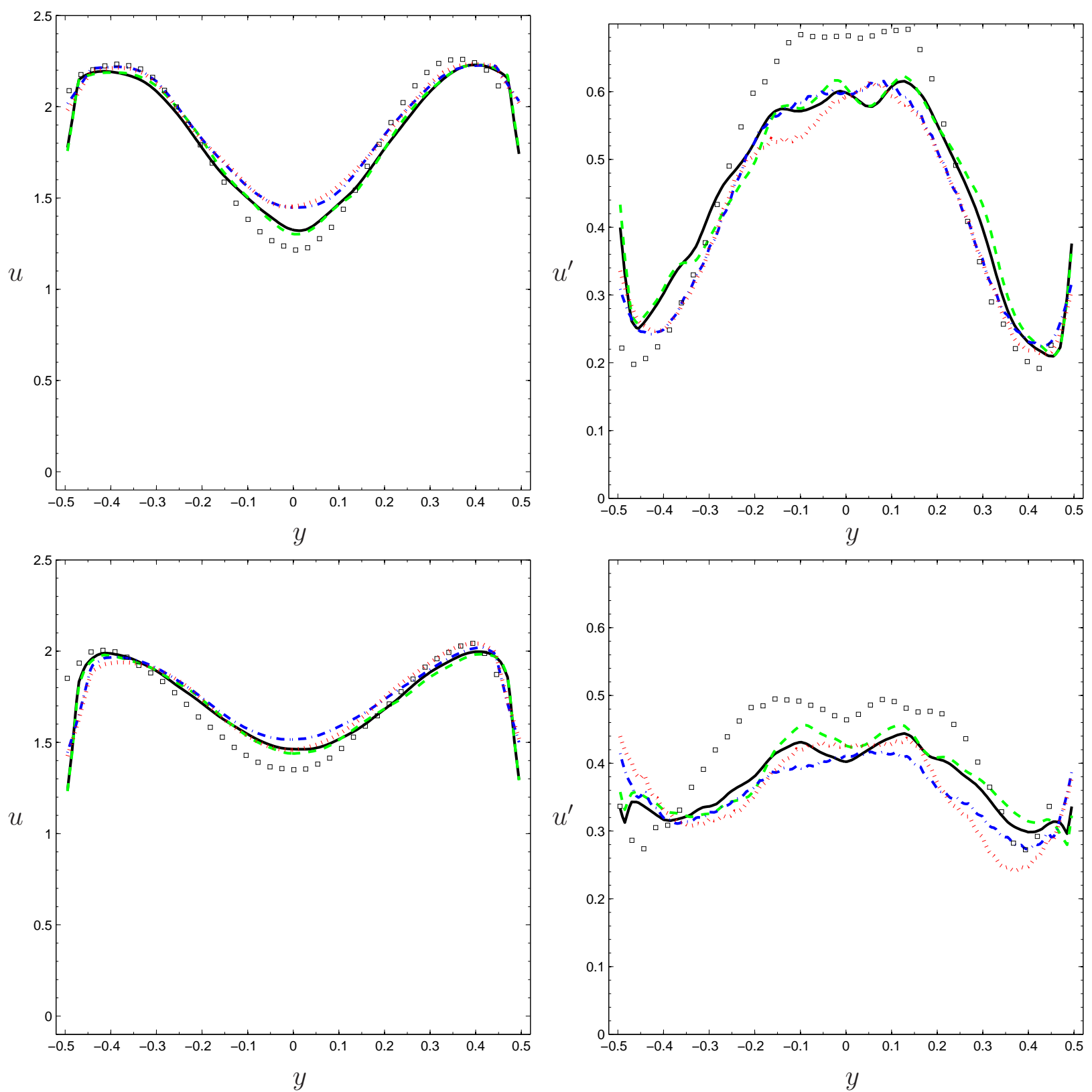

Figure 17. Horizontal profiles of axial mean (left) and rms velocity (right) at $x=1.6$ (top) and $x=2.6$ (bottom) for the experimental data (symbols) and CABARET simulation on a coarser mesh with 0.5 million ponts averaged over a half (red dotted) and full (blue dash-dotted) interval, and on a finer mesh with 4 million points averaged over a half (green dashed) and full (solid black line) time interval. 

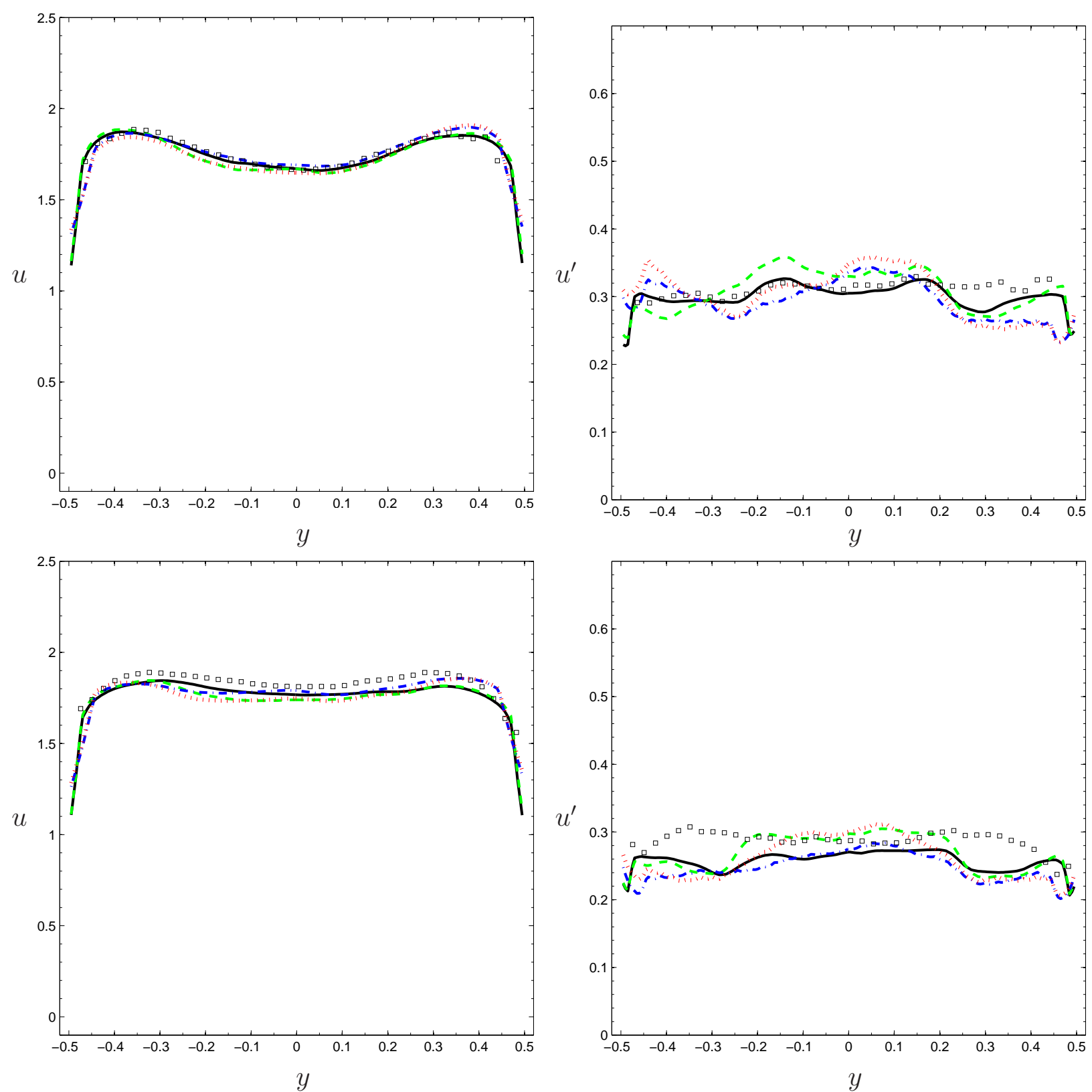

Figure 18. Horizontal profiles of axial mean (left) and rms velocity (right) at $x=3.6$ (top) and $x=4.6$ (bottom) for the experimental data (symbols) and CABARET simulation on a coarser mesh with 0.5 million ponts averaged over a half (red dotted) and full (blue dash-dotted) interval, and on a finer mesh with 4 million points averaged over a half (green dashed) and full (solid black line) time interval. 

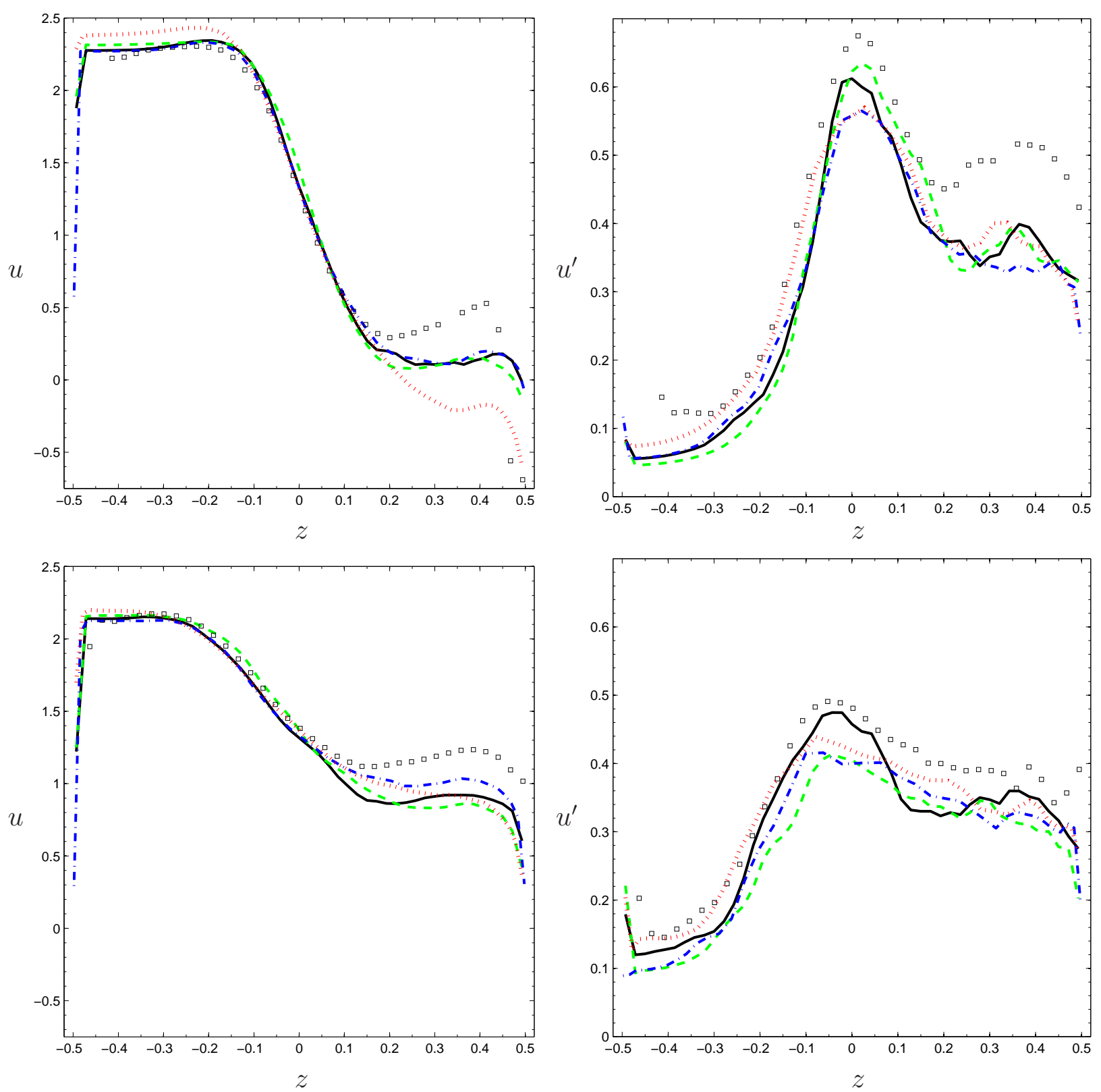

Figure 19. Vertical profiles of axial mean (left) and rms velocity (right) at $x=1.6$ (top) and $x=2.6$ (bottom) for the experimental data (symbols) and Conv3D simulation on 40 milliom node uniform (black solid) and nonuniform (blue dash-dotted) mesh, on 12 million node uniform mesh (green dashed) and on 3 million node nonuniform mesh (red dotted line). 

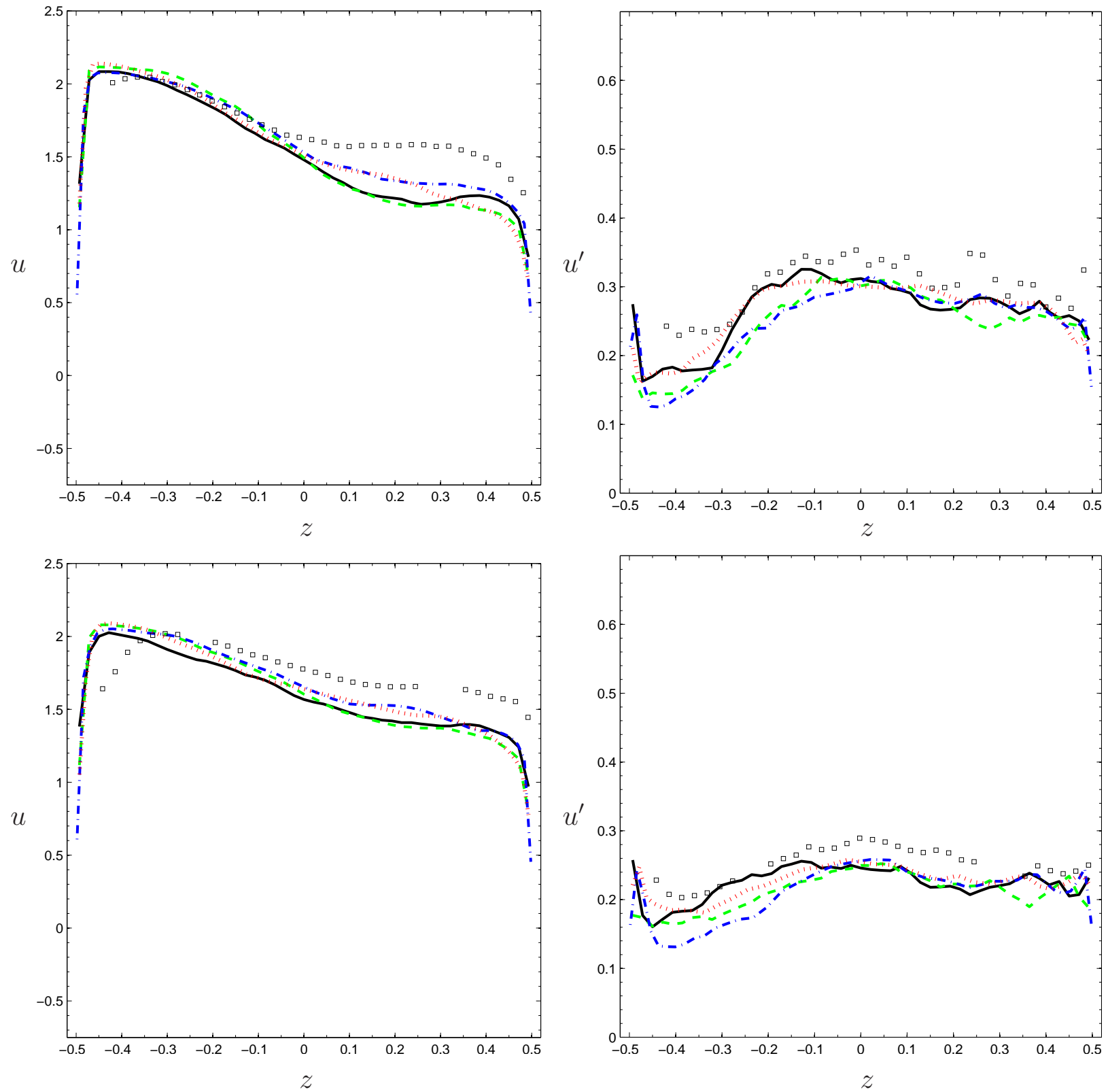

Figure 20. Vertical profiles of axial mean (left) and rms velocity (right) at $x=3.6$ (top) and $x=4.6$ (bottom) for the experiment data (symbols) and Conv3D simulation on 40 milliom node uniform (black solid) and nonuniform (blue dash-dotted) mesh, on 12 million node uniform mesh (green dashed) and on 3 million node nonuniform mesh (red dotted line). 

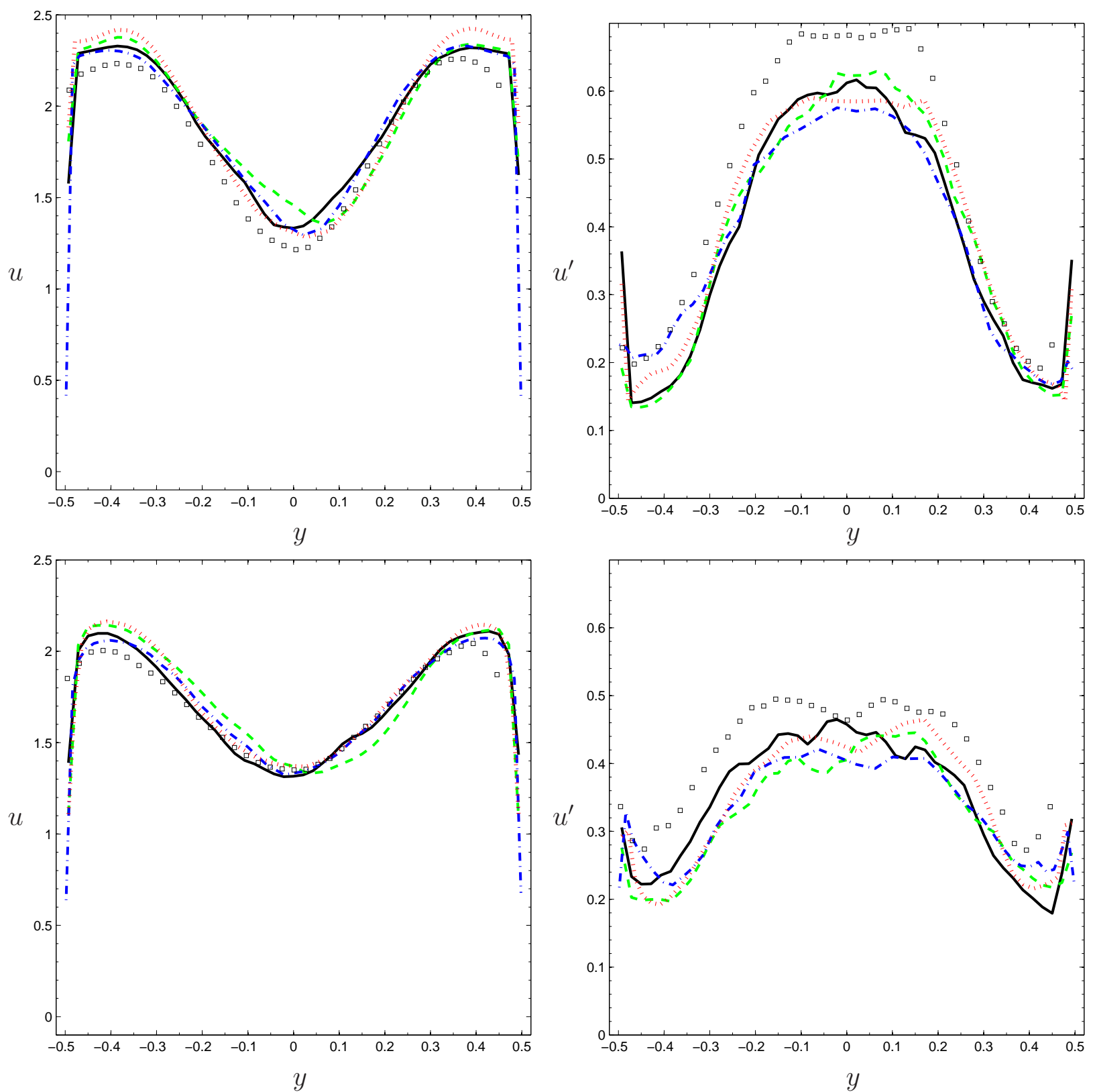

Figure 21. Horizontal profiles of axial mean (left) and rms velocity (right) at $x=1.6$ (top) and $x=2.6$ (bottom) for the experiment data (symbols) and Conv3D simulation on 40 milliom node uniform (black solid) and nonuniform (blue dash-dotted) mesh, on 12 million node uniform mesh (green dashed) and on 3 million node nonuniform mesh (red dotted line). 

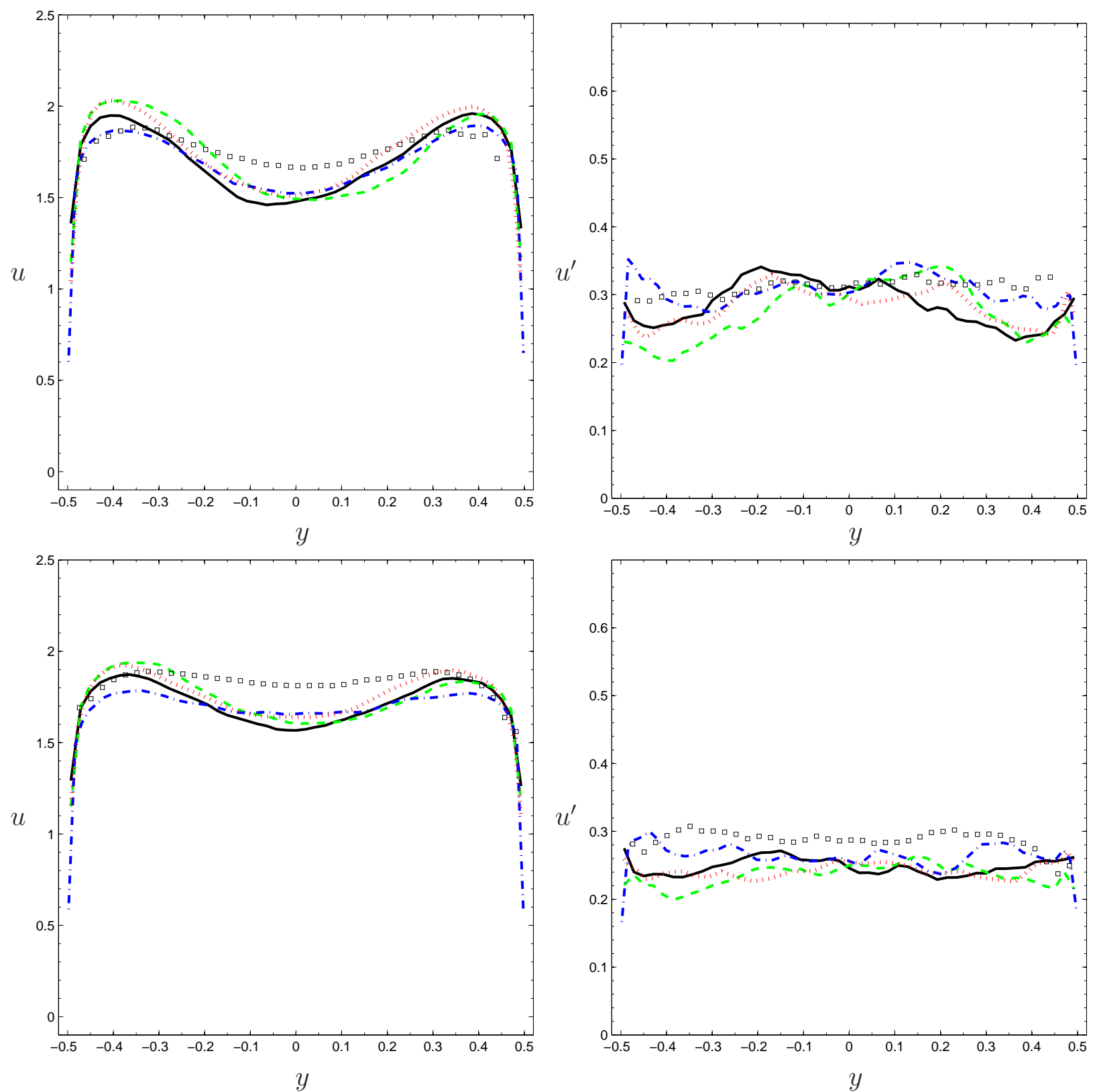

Figure 22. Horizontal profiles of axial mean (left) and rms velocity (right) at $x=3.6$ (top) and $x=4.6$ (bottom) for the experiment data (symbols) and Conv3D simulation on 40 milliom node uniform (black solid) and nonuniform (blue dash-dotted) mesh, on 12 million node uniform mesh (green dashed) and on 3 million node nonuniform mesh (red dotted line). 


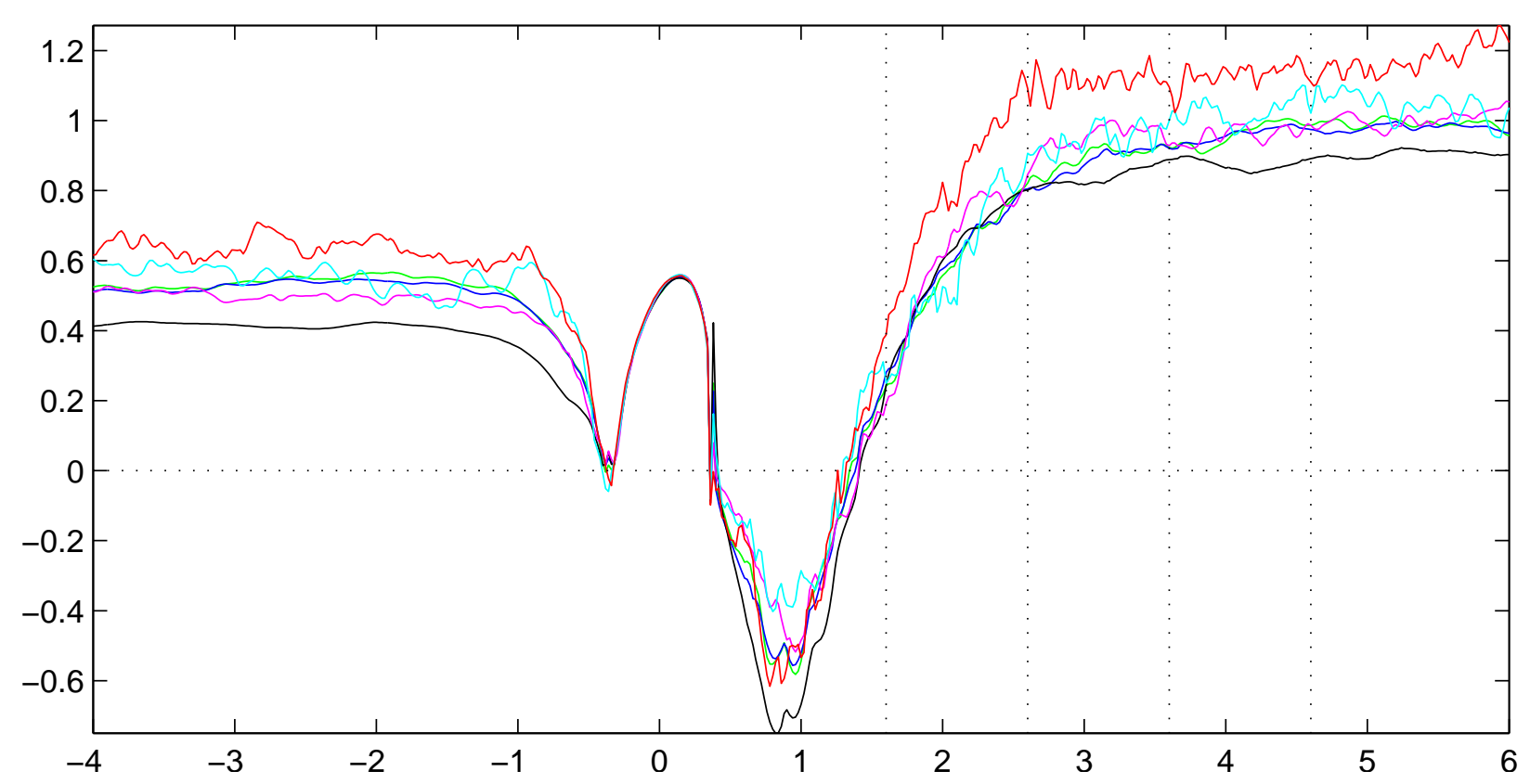

Figure 23. Axial mean velocity profile near the upper wall at $z=0.0050$ and $y=0.0000$ for Nek5000 simulation at $R e=9 \times 10^{4}$ with $N=11$ (red), at $R e=6 \times 10^{4}$ with $N=9$ (cyan) and at $R e=4 \times 10^{4}$ with $N=9$ (magenta), $N=5$ (black) and $N=7$ for the benchmark submission results (green) and longer time averaging (blue). 


\section{Argonne}

\section{Mathematics and Computer Science Division}

Argonne National Laboratory

9700 South Cass Avenue, Bldg. 240

Argonne, IL 60439-4847

www.anl.gov 